\title{
Ginkgo Flavonol Glycosides or Ginkgolides Tend to Differentially Protect Myocardial or Cerebral Ischemia-Reperfusion Injury via Regulation of TWEAK-Fn14 Signaling in Heart and Brain
}

OPEN ACCESS

Edited by:

Francesco Squadrito, University of Messina, Italy

Reviewed by:

Tamer M. A. Mohamed,

University of Louisville, United States

Midori A. Yenari,

University of California,

San Francisco, United States

*Correspondence:

Yan Zhu

yanzhu.harvard@icloud.com

tThese authors have contributed equally to this work.

Specialty section:

This article was submitted to Cardiovascular and Smooth

Muscle Pharmacology,

a section of the journal

Frontiers in Pharmacology

Received: 23 March 2019 Accepted: 07 June 2019

Published: 05 July 2019

Citation:

Xiao G, Lyu M, Wang Y, He S, Liu X,

Ni J, Li L, Fan G, Han J, Gao X, Wang $X$ and Zhu $Y$ (2019)

Ginkgo Flavonol Glycosides or Ginkgolides Tend to Differentially

Protect Myocardial or Cerebral Ischemia-Reperfusion Injury via Regulation of TWEAK-Fn14 Signaling in Heart and Brain

Front. Pharmacol. 10:735. doi: 10.3389/fphar.2019.00735
Guangxu Xiao ${ }^{1,2 \dagger}$, Ming Lyu ${ }^{1,2,3 \dagger}$, Yule Wang ${ }^{1,2}$, Shuang He $\mathrm{H}^{1,2}$, Xinyan Liu ${ }^{1,2}$, Jingyu Ni ${ }^{1,4}$, Lan Li ${ }^{1,4}$, Guanwei Fan ${ }^{4}$, Jihong Han ${ }^{5}$, Xiumei Gao ${ }^{1}$, Xiaoying Wang ${ }^{1,6}$ and Yan Zhu ${ }^{1,2 *}$

\footnotetext{
${ }^{1}$ Tianjin State Key Laboratory of Modern Chinese Medicine, Tianjin University of Traditional Chinese Medicine, Tianjin, China, ${ }^{2}$ Research and Development Center of TCM, Tianjin International Joint Academy of Biotechnology \& Medicine, Tianjin, China, ${ }^{3}$ Institute of Chinese Materia Medica, China Academy of Chinese Medicial Sciences, Beijing, China, ${ }^{4}$ Medical Experiment Center, First Teaching Hospital of Tianjin University of Traditional Chinese Medicine, Tianjin, China, ${ }^{5}$ College of Life Sciences, Key Laboratory of Medicinal Chemical Biology, Key Laboratory of Bioactive Materials of Ministry of Education, Nankai University, Tianjin, China; College of Biomedical Engineering, Hefei University of Technology, Hefei, China, ${ }^{6}$ Neuroprotection Research Laboratory, Departments of Radiology and Neurology, Massachusetts General Hospital, Harvard Medical School, Charlestown, MA, United States
}

Shuxuening injection (SXNI), one of the pharmaceutical preparations of Ginkgo biloba extract, has significant effects on both ischemic stroke and heart diseases from bench to bedside. Its major active ingredients are ginkgo flavonol glycosides (GFGs) and ginkgolides (GGs). We have previously reported that SXNI as a whole protected ischemic brain and heart, but the active ingredients and their contribution to the therapeutic effects remain unclear. Therefore, we combined experimental and network analysis approach to further explore the specific effects and underlying mechanisms of GFGs and GGs of SXNI on ischemia-reperfusion injury in mouse brain and heart. In the myocardial ischemiareperfusion injury (MIRI) model, pretreatment with GFGs at $2.5 \mathrm{ml} / \mathrm{kg}$ was superior to the same dose of GGs in improving cardiac function and coronary blood flow and reducing the levels of lactate dehydrogenase and aspartate aminotransferase in serum, with an effect similar to that achieved by SXNI. In contrast, pretreatment with GGs at $2.5 \mathrm{ml} / \mathrm{kg}$ reduced cerebral infarction area and cerebral edema similarly to that of SXNI but more significantly compared with GFGs in cerebral ischemia-reperfusion injury (CIRI) model. Network pharmacology analysis of GFGs and GGs revealed that tumor necrosis factorrelated weak inducer of apoptosis (TWEAK)-fibroblast growth factor-inducible 14 (Fn14) signaling pathway as an important common mechanism but with differential targets in MIRI and CIRI. In addition, immunohistochemistry and enzyme linked immunosorbent assay (ELISA) assays were performed to evaluate the regulatory roles of GFGs and GGs on the common TWEAK-Fn14 signaling pathway to protect the heart and brain. Experimental results confirmed that TWEAK ligand and Fn14 receptor were downregulated by GFGs to mitigate MIRI in the heart while upregulated by GGs to improve CIRI in the brain. In 
conclusion, our study showed that GFGs and GGs of SXNI tend to differentially protect brain and heart from ischemia-reperfusion injuries at least in part by regulating a common TWEAK-Fn14 signaling pathway.

Keywords: Shuxuening injection, ginkgo flavonol glycosides, ginkgolides, myocardial ischemia-reperfusion injury, cerebral ischemia-reperfusion injury, TWEAK-Fn14 signaling

\section{INTRODUCTION}

Cardio-cerebral vascular diseases, including mainly ischemic myocardial infarction and ischemic stroke, are the most common causes of disability and death worldwide (Benjamin et al., 2018). It is well-established that ischemia-reperfusion (I/R) injury of both heart and brain shares certain common pathological mechanisms such as inflammation (Iadecola and Anrather, 2011; Goldfine and Shoelson, 2017), oxidative stress (Sanderson et al., 2013; Muntean et al., 2016), microvascular dysfunction (Gursoy-Ozdemir et al., 2012; Granger and Kvietys, 2017), and mitochondrial dysfunction (Ago et al., 2010; Yang et al., 2015). Recently, increasing numbers of research have explored underlying mechanisms of the intimate internal connection between ischemic stroke and heart diseases (Prosser et al., 2007; Samuels, 2007; Ishikawa et al., 2013; Abete et al., 2014; Gladstone et al., 2014; Sanna et al., 2014; Chen et al., 2017c; Lyu et al., 2017; Lyu et al., 2018). In recent years, a growing number of studies indicated that certain traditional Chinese medicines (TCMs) and their main active ingredients have protective and curative effects on I/R-mediated injury in brain and heart (Han et al., 2017).

Extracts of Ginkgo biloba (EGB) is one of the most common and earliest industrialized herbal medicinal preparations. As a pharmaceutical brand of EGB, Shuxuening injection (SXNI) is approved by the Sino Food and Drug Administration, and its major identified active ingredients consist of ginkgo flavonol glycosides (GFGs) and ginkgolides (GGs). EGB has a broad range of pharmacological effects, such as anti-inflammation (Chen et al., 2017b), antioxidant (Saini et al., 2014), anti-apoptotic (Yu et al., 2018), antidepressant ( $\mathrm{Hu}$ et al., 2018), anti-arrhythmia (Zhao et al., 2013), antitumor (Li et al., 2017), improving cognitive function (Wan et al., 2016), and relieving I/R injury (Jin et al., 2014; Ran et al., 2014). Most recently, its therapeutic effect on cardio-cerebrovascular diseases has attracted renewed attention (Yang et al., 2017). Meanwhile, research on GFGs and GGs is deepening. So far, identified GFGs include quercetin, kaempferol, myricetin, apigenin, isorhamnetin, luteolin, and tamarixetin, whereas identified GGs consist of ginkgolide A, ginkgolide B, ginkgolide $\mathrm{C}$, ginkgolide J, ginkgolide $\mathrm{M}$, ginkgolide $\mathrm{K}$, ginkgolide $\mathrm{L}$, ginkgolide $\mathrm{P}$, ginkgolide $\mathrm{Q}$, and bilobalide (van Beek and Montoro, 2009; Liao et al., 2011; Mohanta et al., 2014). Taking quercetin in GFGs as an example, its variety of pharmacological activities include anti-inflammatory (Song et al., 2018), antioxidation (Fadda et al., 2018), anticancer (Patra et al., 2018), anti-infective (Mehrbod et al., 2018), hypotensive (Marunaka et al., 2017), and hypoglycemic (Zhuang et al., 2018). The role of quercetin in the treatment of cardiovascular diseases and neurodegenerative disease is also frequently reported (Enogieru et al., 2018; Patel et al., 2018). The pharmacological effects of GGs are also extensive, mainly including anti-apoptotic (Guo et al., 2015), neuroprotection (Zheng et al., 2016), antioxidative stress (Priyanka et al., 2014), anti-arrhythmia (Zhao et al., 2013), and antiplatelet (Rui et al., 2016). Consequently, more attention has been drawn to studying the underlying active ingredients and molecular mechanisms of SXNI in preventing and treating ischemia stroke and coronary heart disease.

Tumor necrosis factor (TNF)-related weak inducer of apoptosis (TWEAK), also named as TNFSF12, APO3L, or CD255, is a member of the TNF superfamily (Chicheportiche et al., 1997; Marsters et al., 1998), while fibroblast growth factorinducible 14 (Fn14), also named as TNFRSF12A, TWEAKR, or CD266 (Meighan-Mantha et al., 1999; Wiley et al., 2001), is the only known TWEAK receptor (Bossen et al., 2006). TWEAKFn14 axis has shown an increasingly important role in cardiocerebral vascular diseases (Blanco-Colio, 2014). The interaction of TWEAK and Fn14 activates downstream signaling processes during disease development and progression, which includes mediating atrial-derived HL-1 myocytes hypertrophy via the JAK2/STAT3 signaling pathway (Hao et al., 2018), weakening the antiproliferative effects of miR-149 in osteosarcoma via the AKT serine/threonine kinase (PI3K/AKT) signaling pathway (Xu et al., 2018), inducing pro-fibrotic responses leading to heart failure via the NF- $\kappa \mathrm{B}$ and/or AP-1 signaling pathway (Das et al., 2018), preventing renal damage in patients with lupus nephritis via type I interferon signaling pathway (Xue et al., 2017), and promoting wound healing processes through favoring regional inflammation, cytokine production, and extracellular matrix synthesis (Liu et al., 2018). In addition, TWEAK also participates in a variety of other diseases such as tumors (Hu et al., 2017), neonatal hypoxiaischemia (Kichev et al., 2018), human glioma (Guan et al., 2017), psoriasis (Sidler et al., 2017), and chronic colitis (Son et al., 2013).

We have previously reported that SXNI reduces both myocardial and cerebral I/R injuries in heart and brain via a common protection mechanism involving Tnfrsf12a-mediated atherosclerosis signaling and inflammatory response (Lyu et al., 2018). However, since SXNI contains multicomponents, the specific contribution by different ingredients and their pharmacological mechanisms remain poorly understood in this setting. In the present study, we deciphered the roles of GFGs and GGs and confirmed that GFGs were superior than GGs in exhibiting the protective effect on myocardial I/R injury via suppressing TWEAK-Fn14 signaling and GGs protected cerebral I/R injury more effectively compared with GFGs by promoting TWEAK-Fn 14 signaling. Our new findings may contribute to further understanding of the organ-specific therapeutic effects of SXNI and provide a new basis for the clinical application 
of EGBs, which will also promote the development and application in TCM.

\section{MATERIALS AND METHODS}

\section{Drugs and Reagents}

SXNI (drug approval number: Z13020795; batch number: 15101201), GFGs, and GGs were supplied by China Shineway Pharmaceutical Group Ltd. (Shijiazhuang, China). According to manufacturer's instruction, the chemical content of one SXNI dose ( $5 \mathrm{ml} /$ unit) is equal to $17.5 \mathrm{mg}$ Ginkgo biloba leaf extract, which includes $4.2 \mathrm{mg}$ total GFGs and $0.7 \mathrm{mg} \mathrm{GGs}$. GFGs $(0.84 \mathrm{mg} / \mathrm{ml})$ and GGs $(0.14 \mathrm{mg} / \mathrm{ml})$ are also supplied as injections (Ma et al., 2018). We bought 2, 2, 2-Tribromoalcohol from Sigma (T48402, St. Louis, MO, United States). High-efficiency radioimmunoprecipitation assay tissue/cell lysate (R0010), 2, 3, 5-Triphenyl-2H-tetrazolium chloride (TTC, T8170), and bicinchoninic acid (PC0020) protein concentration assay kit were purchased from Solarbio (Beijing, China). Anti-TWEAKR antibody was purchased from Abcam (EPR3179, Shanghai, China). Mouse ELISA kits for TWEAK, interleukin 6, and annexin $\mathrm{A} 1$ were purchased from $\mathrm{ZCi} \mathrm{BiO}$ (Shanghai, China). Other ELISA kits for lactate dehydrogenase $(\mathrm{LDH})$, aspartate aminotransferase (AST), creatine kinase-MB (CK-MB), and creatine kinase (CK) were purchased from Biosino Bio-Technology and Science Inc. (Beijing, China).

\section{Animals}

Male 6-week-old C57BL/6J mice (22 \pm 2 g) were purchased from Beijing Vital River Laboratory Animal Technology Co., Ltd. (Beijing, China, Certificate no.: SCXK Jing 2016-0006). This study was carried out in accordance with the principles of the Basel Declaration and recommendations of the Care and Use of Laboratory Animals issued by the Ministry of Science and Technology of China. The protocol was approved by the Laboratory Animal Ethics Committee of Tianjin University of TCM (Permit Number: TCM-LAEC2014004). The experimental animals were previously randomized into several groups and kept with commercial rat food and water. They were housed under controlled temperature $\left(22^{\circ} \mathrm{C}\right)$ and relative humidity $(40 \% \pm 5 \%)$ conditions with a 12 -h light/ dark cycle.

\section{Establishment of Myocardial Ischemia- Reperfusion Injury Model and Drug Administration}

A mouse model of myocardial I/R injury (MIRI) was performed as previously described (Gao et al., 2010). Briefly, mice were anesthetized by inhalation of $3 \%$ isoflurane driven by $100 \% \mathrm{O}_{2}$ in an induction chamber and then moved to an operating table in a supine position and kept under anesthesia using an inhalation mask and $2 \%$ isoflurane $\left(100 \mathrm{ml} / \mathrm{min} \mathrm{O}_{2}\right)$. Next, the chest of mouse was opened at the intercostal space between 3 and 4 sternal rib via a left thoracotomy. Once the heart was squeezed out, the proximal left anterior descending coronary artery under left auricle is transiently ligated by a slipknot utilizing a 7-0 silk suture. Following the thorax closure and as soon as spontaneous respiration was sufficient, the mice were released and placed on an electric blanket. After $30 \mathrm{~min}$ of ischemia, the slipknot was untied and followed by $24 \mathrm{~h}$ of reperfusion. ST-segment elevation on an electrocardiogram monitor represented a success in MIRI model surgery. In sham control mice, the surgical procedures were identical, except the left anterior descending coronary artery was not tied. The mice were stochastically divided into five groups in the following experiments: sham, I/R (saline), I/R+SXNI (dose, $2.5 \mathrm{ml} / \mathrm{kg}$ ), I/R+GFGs (dose, $2.5 \mathrm{ml} / \mathrm{kg}$ ), and I/R+GGs (dose, $2.5 \mathrm{ml} / \mathrm{kg}$ ). SXNI, GFGs, GGs, and saline were administered via intravenous tail injection within 10 min after the onset of ischemia. Sham and I/R groups were intravenously administered with $0.9 \%$ normal saline. No differences in operative mortality were observed among groups investigated.

\section{Establishment of Cerebral Ischemia- Reperfusion Injury Model and Drug Administration}

For preparation of cerebral I/R injury (CIRI) model (Rousselet et al., 2012), anesthesia was induced with $2 \%$ isoflurane inhalation and then maintained with $1.5 \%$ isoflurane using a small animal respirator (RWD, Inc., China). After a midline incision at the neck, the right common carotid artery, internal carotid artery (ICA), and external carotid artery (ECA) were exposed via careful blunt separation, and the occipital, the cranial thyroid, the pterygopalatine artery, and the ascending pharyngeal artery were cauterized utilizing a monopolar electrical cautery. After occlusion of the right common carotid artery and ICA using two micro-clips, the right distal ECA was tightly tied by two straps using a sterile 6-0 silk suture and interrupted from the middle. Then, a small hole was made in the right ECA utilizing a micro-scissor, and a silicone-coated 4-0 nylon monofilament (Jialing Biotechnology Co., Ltd., Guangzhou, China) was introduced into the right ECA and gently advanced through the right ICA until its tip blocked the origin of the right middle cerebral artery (MCA), leading a decline of local cortical blood flow in the right MCA territory to $20-30 \%$ of baseline. After 60 min of ischemia, the blood flow was restored by allowing the filament withdrawal and followed by $24 \mathrm{~h}$ of reperfusion. Shamoperated mice were operated identically except for the MCA occlusion procedure. The mice were then randomly divided into five groups: sham, I/R (saline), I/R+SXNI (dose, $2.5 \mathrm{ml} /$ $\mathrm{kg}$ ), I/R+GFGs (dose, $2.5 \mathrm{ml} / \mathrm{kg}$ ), and I/R+GGs (dose, $2.5 \mathrm{ml} /$ $\mathrm{kg}$ ). Drug and saline were injected through the vein after 50 min of ischemia.

\section{Echocardiographic Measurement}

The noninvasive assessment of left ventricular function and coronary blood flow in the heart were made using an ultrahigh resolution small animal ultrasound imaging system (Vevo 2100, VisualSonics, Toronto, ON, Canada) equipped with a 
30-MHz transducer (Chen et al., 2016; Li et al., 2016). Mice were anesthetized by inhaling $1.5-2.0 \%$ isoflurane and transferred to the dorsal position and placed on a heated imaging platform. Parameters indicating cardiac functions were measured by M-mode and color Doppler mode as follows: left ventricular (LV) ejection fraction (EF), LV fractional shortening (FS), cardiac output (CO), stroke volume, LV internal dimensions at diastole (LVIDd), LV internal diameter systole (LVIDs), LV posterior wall diastole (LVPWd), LV posterior wall systole (LVPWs), LV systole volume (LV Vols), LV diastole volume (LV Vold), heart rate and LV mass, along with aortic valve (AV) peak velocity, AV peak pressure, and aorta velocity-time integral mean velocity (AoV VTI).

\section{Hemodynamic Evaluation of Left Ventricular Function}

After $24 \mathrm{~h}$ of reperfusion, the cannulation connecting the biofunction experiment system (MP100-CE, BIOPAC Systems, Inc., United States) was inserted into the left ventricle through right carotid artery (Chen et al., 2015; Wang et al., 2017). Left ventricular maximum upstroke velocity $\left(+\mathrm{dp} / \mathrm{dt}_{\max }\right)$ and left ventricular maximum descent velocity $\left(-\mathrm{dp} / \mathrm{dt}_{\max }\right)$ were measured to reflect and evaluate the left ventricular systolic and diastolic function.

\section{Measurement of Biochemical Parameters}

At $24 \mathrm{~h}$ after myocardial ischemia and reperfusion, blood was collected, and serum was separated by centrifugation at 3,000 rpm for 15 min. Concentrations of LDH, AST, CK-MB, and CK in mouse plasma were measured by ELISA kits in automatic biochemical analyzer (Multiskan MK3; Thermo Fisher Scientific, Waltham, MA, United States) according to the manufacturer's instructions. These biochemical parameters, commonly used in clinical diagnostics to detect myocardial inflammation and acute myocardial infarction, were used to evaluate the myocardial damage in this research.

\section{Hematoxylin and Eosin Staining}

After collecting the blood samples, the myocardial tissues were rapidly excised, rinsed in $0.9 \%$ saline, and fixed in $10 \%$ paraformaldehyde solution for more than $48 \mathrm{~h}$. Heart tissues were then cut into four 5-mm-thick slices perpendicular to the long axis of the heart and dehydrated in the automatic dehydrator (Excelsior, Thermo., Ltd., United States) with a following program: $70 \%$ alcohol for $1 \mathrm{~h}, 80 \%$ alcohol for $1 \mathrm{~h}, 90 \%$ alcohol for $1 \mathrm{~h}, 95 \%$ alcohol for $1 \mathrm{~h}$, anhydrous alcohol I for $1 \mathrm{~h}$, anhydrous alcohol II for $1 \mathrm{~h}$, xylene I for $40 \mathrm{~min}$, xylene II for $40 \mathrm{~min}$, xylene III for $40 \mathrm{~min}$, paraffin I for $2 \mathrm{~h}$, paraffin for II $2 \mathrm{~h}$, and paraffin III for $2 \mathrm{~h}$. At the end of the procedure, the tissues were removed and embedded in paraffin. The paraffin blocks were pruned and cut into serial slices of 5-mm thick using a manual slicer (HM355S, Thermo., Ltd., United States), which were then placed into the automatic dyeing machine (Gemini, Thermo., Ltd., United States) for staining. The following staining routine was compiled: tissue dewaxing liquid I for $5 \mathrm{~min}$, tissue dewaxing liquid II for $5 \mathrm{~min}$, tissue dewaxing liquid III for $5 \mathrm{~min}$, anhydrous alcohol for $5 \mathrm{~min}$, anhydrous alcohol for $3 \mathrm{~min}, 95 \%$ alcohol for $1 \mathrm{~min}, 85 \%$ alcohol for $1 \mathrm{~min}, 75 \%$ alcohol for $1 \mathrm{~min}$, acid alcohol for $5 \mathrm{~s}$, running water wash for $1 \mathrm{~min}$, hematoxylin for $7 \mathrm{~min}$, running water wash for $1 \mathrm{~min}$, acid alcohol for $5 \mathrm{~s}$, running water wash for $1 \mathrm{~min}$, bluing reagent for $30 \mathrm{~s}$, running water wash for $1 \mathrm{~min}$, aqueous eosin for $3 \mathrm{~min}$, running water wash for $1 \mathrm{~min}, 75 \%$ alcohol for $30 \mathrm{~s}, 85 \%$ alcohol for $30 \mathrm{~s}, 95 \%$ alcohol for $30 \mathrm{~s}, 100 \%$ alcohol for $1 \mathrm{~min}, 100 \%$ alcohol for $1 \mathrm{~min}$, xylene I for $1 \mathrm{~min}$, xylene II for 1 min, and xylene III for $1 \mathrm{~min}$. After the sections were mounted by using automatic sealing machine (ClearVue, Thermo., Ltd., United States), myocardial tissues of pathological changes were observed by optical microscope (DP71, OLYMPUS, Japan).

\section{Quantitative Measurement of Cerebral Infarct Size}

At $24 \mathrm{~h}$ after reperfusion, brains of euthanized mice were removed. The cerebral tissues were crosscut into five pieces of 2 -mm-thick slices in a brain matrix device, which were added to the $2 \%$ solution of TTC in $0.1 \mathrm{M}$ phosphate buffered solution (PBS), incubated at $37^{\circ} \mathrm{C}$ in dark for $10 \mathrm{~min}$. After staining by TTC, the sections were photographed and used to measure the cerebral infarct size using ImageJ analysis software (National Institutes of Health, Bethesda, MD, United States) and calculated as the proportion of cerebral infarct size (percentage) $=$ infarct size (while pale area)/total area of transverse slice $\times 100 \%$.

\section{Analysis of Edema Volume}

Anesthetized mice were injected with arterial angiography agent (Iohexol, $15 \mathrm{ml} / \mathrm{kg}$ ) through the tail vein. Next, the mouse brain was imaged with a $\mu \mathrm{CT}$ small animal imager (Quantum FX; PerkinElmer, United States) with the following imaging parameters: voltage for $90 \mathrm{kV}$, current for $180 \mu \mathrm{A}$, field of view for $20 \mathrm{~mm}$, scan technique for std $4.5 \mathrm{~min}, 360$ views. The severity of cerebral edema was evaluated using ImageJ analysis software (National Institutes of Health, Bethesda, MD, United States) to calculate the offset distance of midline in the coronal image of the mouse brain at Bregma $0.3 \mathrm{~mm}$ (Park et al., 2014). The greater the offset distance of the midline, the more serious the cerebral edema.

\section{Neurological Deficit Score}

At $24 \mathrm{~h}$ after reperfusion, according to the Bederson scale (Bederson et al., 1986) to assess the neurological deficit of mice, the neurological deficit score table is as follows: 0, walking normally, no observable defects; 1 , forelimb flexion; 2 , decreased resistance to lateral push without circling; 3 , circling behavior in addition to the former symptoms; 4 , ambulation difficulty or inability.

\section{Enzyme-Linked Immunosorbent Assay}

After collecting the plasma of MIRI and CIRI mice, the hearts and brains were removed separately. The plasma was centrifuged at $1,000 \mathrm{r} / \mathrm{min}$ for $15 \mathrm{~min}$ to separate the serum. Fresh tissues 
of heart and brain were smashed using ultrasonic cell pulverizer (SCIENTZ-II D, Xinzhi Biotechnology Co., Ltd., China) and added the high-efficiency radioimmunoprecipitation assay tissue/cell lysate to collect the supernatant of tissue homogenates. TWEAK protein concentration in supernatant was determined using an ELISA kit (ZC-54137, ZCi Biotechnology Co., Ltd). according to the manufacturer's instructions.

\section{Immunohistochemistry}

The paraffin sections of heart and brain tissues (5- $\mu$ m thick) were deparaffinized, incubated in distilled water, rinsed $(3 \times 5 \mathrm{~min})$ in PBS-T (0.01 M PBS pH 7.4: $\mathrm{KH}_{2} \mathrm{PO}_{4} 0.02 \%$, $\mathrm{N}_{2} \mathrm{HPO}_{4} 0.29 \%, \mathrm{KCl} 0.02 \%, 0.8 \% \mathrm{NaCl}, 0.05 \%$ bovine serum albumin (BSA), Tween-20 0.05\%, 0.0015\% TritonX-100), and then blocked endogenous peroxidase via incubating in 3\% peroxide-methanol at room temperature for $10 \mathrm{~min}$. After incubation for $1 \mathrm{~h}$ in blocking buffer (10\% bovine serum albumin), the sections were stained for $2 \mathrm{~h}$ at $37^{\circ} \mathrm{C}$ using anti-TWEAKR antibody diluted with blocking buffer (1:50). Following wash in PBS-T $(3 \times 5 \mathrm{~min})$, the sections were incubated sequentially for $1 \mathrm{~h}$ with each of the corresponding secondary antibody (Biotinylated Goat anti-Rabbit IgG) and the third antibody (Horseradish Peroxidase Streptavidin) at $37^{\circ} \mathrm{C}$. The immunoreactivity of TWEAKR protein was visualized using 3, 3'-diaminobenzidine tetrahydrochloride hydrate. Finally, counterstaining was performed with hematoxylin, dehydrated, and mounted. The expression of TWEAKR (Fn14) was detected by optical microscope (DP71, OLYMPUS, Japan). The expression of Fn 14 is quantified by calculating its integrated optical density value using ImagePro Plus software (National Institutes of Health, Bethesda, MD, United States) (Pei et al., 2013).

\section{Network Pharmacology Analysis}

In previous studies, we have identified the significantly differentially expressed genes after SXNI treatment in mice of MIRI and CIRI. Database of GFGs and GGs were established via literature mining. In addition, the corresponding targets of the compounds were obtained from the integration of Ingenuity $^{\circledR}$ Pathway Analysis (IPA), Traditional Chinese Medicine Systems Pharmacology Database and Analysis Platform, Traditional Chinese Medicine Integrative Database for Herb Molecular Mechanism Analysis, and other databases. Then, the above data were submitted to Ingenuity ${ }^{\circledR}$ Pathway Analysis (IPA) to execute "Build-Path Explorer" module to determine the crucially target genes of GFGs and GGs.

\section{Statistical Analysis}

Experimental data were presented as the mean \pm SEM or mean \pm SD. The GraphPad Prism 7 software (GraphPad Software, Inc., La Jolla, CA, United States) was utilized for statistical analysis, which was performed with Student's two-tailed $t$-test for comparison between two groups or one-way ANOVA for multiple groups. A value of $P<0.05$ was defined as statistically significant.

\section{RESULTS}

\section{Ginkgo Flavonol Glycosides Are Superior Than Ginkgolides in Improving Cardiac Function in Myocardial Ischemia- Reperfusion Injury Mice}

The effects of GFGs and GGs on cardiac function were first examined via performing the echocardiography. The I/R group significantly decreased LVEF \%, LVFS \%, CO, stroke volume, and LVPWs, while the sham group had increased LVIDd, LVIDs, LV Vold, and LV Vols. No significant change was observed for LVPWd, heart rate, and LV mass (Figure 1). However, the marked increase in LVEF \% (Figure 1B), LVFS \% (Figure 1C), $\mathrm{CO}$ (Figure 1D), and stroke volume (Figure 1E) and decrease in LVIDd (Figure 1F), LVIDs (Figure 1B), LV Vold (Figure 1J), and LV Vols (Figure 1K) were significantly reversed by SXNI and GFGs treatment. By contrast, only decreased LVIDd (Figure 1F), LV Vold (Figure 1J), and LV Vols were observed in the I/R+GGs group (Figure 1K). GFGs, GGs, or SXNI had no effect on LVPWd (Figure 1H), LVPWs (Figure 1I), heart rate (Figure 1L), and LV mass (Figure 1M) compared with the I/R group. In addition, the effects of GFGs and GGs on coronary blood flow were obtained in color Doppler mode at $18 \mathrm{~h}$ after myocardial I/R (Figure 2A). As the picture shows, the decline in AoV VTI (Figure 2B), AV peak velocity (Figure 2C), AV peak pressure (Figure 2D), left ventricular maximum upstroke velocity $\left(+\mathrm{dp} / \mathrm{dt}_{\max }\right)$ (Figure $\left.2 \mathrm{E}\right)$, and left ventricular maximum descent velocity $\left(-\mathrm{dp} / \mathrm{dt}_{\max }\right.$ ) (Figure $2 \mathrm{~F}$ ) in the $\mathrm{I} / \mathrm{R}$ group were outstanding compared with the sham group. However, GFG and SXNI treatment significantly reversed this decline compared with GGs (Figure 2E and F). Taken together, these data indicate that the therapeutic effect of GFGs is superior to GGs in improving cardiac function in MIRI mice.

\section{Ginkgo Flavonol Glycosides Are Superior to Ginkgolides in Attenuating Myocardial Injury in Myocardial Ischemia-Reperfusion Injury Mice}

After 30-min ischemia and 24-h reperfusion, the protective effects of GFGs and GGs on myocardial injury in MIRI mice were investigated via observing the histopathological features of the myocardial tissue and detecting the levels of LDH, AST, $\mathrm{CK}-\mathrm{MB}$, and $\mathrm{CK}$ in serum. The results indicated that the elevated levels of LDH and AST caused by I/R injury were significantly reduced by GFGs and SXNI administration (Figures 3 B and C). Furthermore, SXNI also obviously decreased the level of CK in serum (Figure 3D). On the other hand, GGs only marginally altered serum AST compared with the I/R group (Figure 3C). The results of the hematoxylin and eosin staining showed that $I / R$ injury led to extensive myocardial structural disarray, increased necrosis and fusion area, as well as infiltration of inflammatory cells into myocardial tissue, which were indicated with yellow arrows (Figure 3A). However, GFGs and SXNI were significantly more effective than GGs in improving the histological features of 


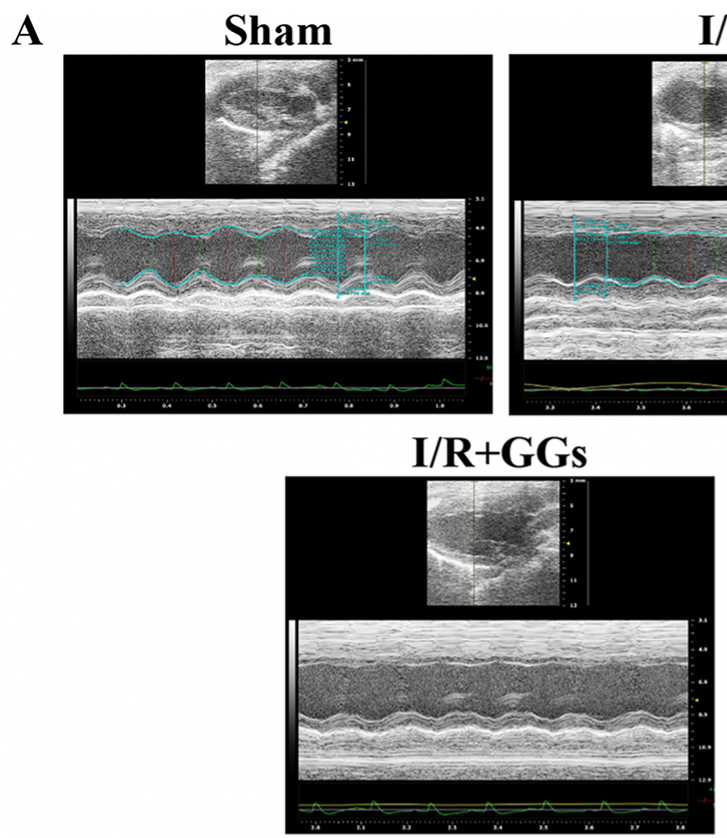

I/R
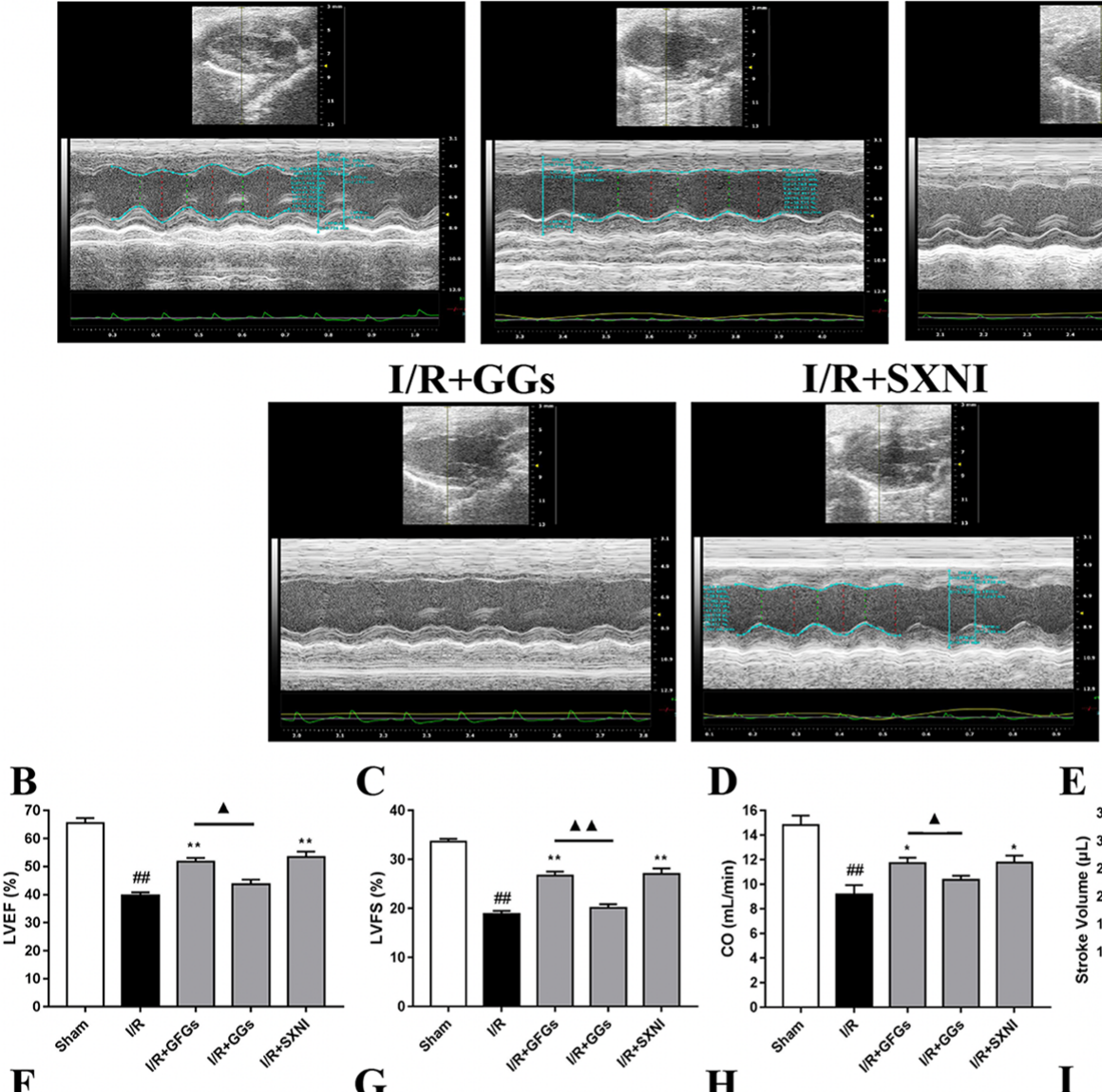

C

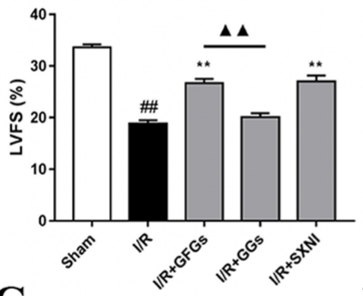

G
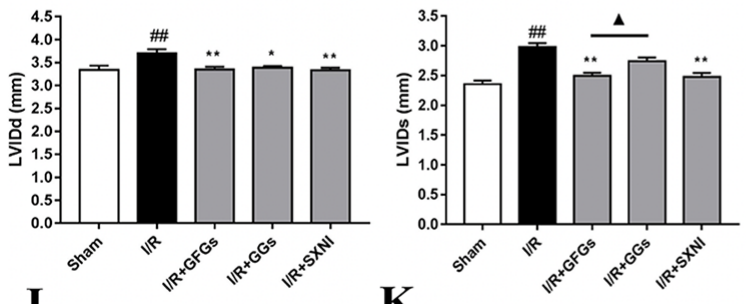

K
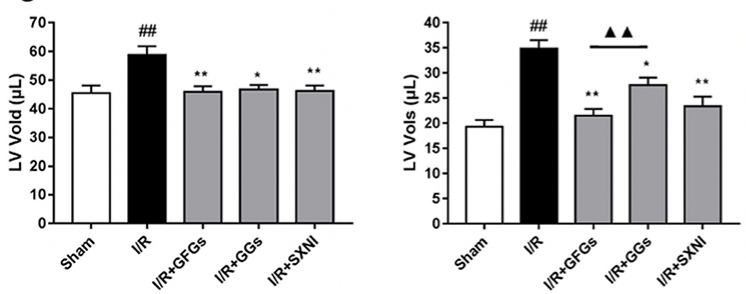

D

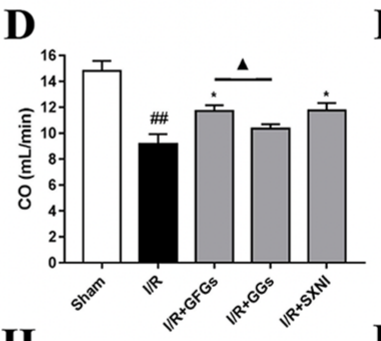

H

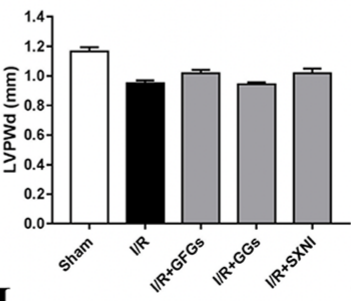

L

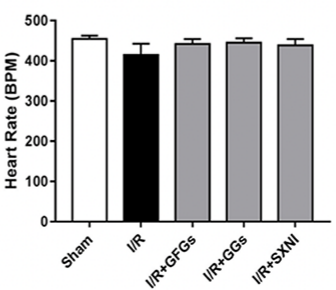

E

I
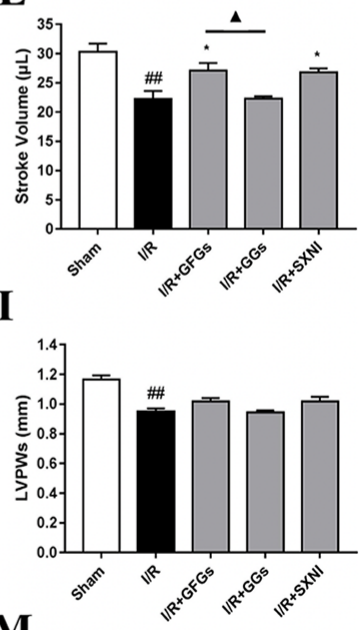

M

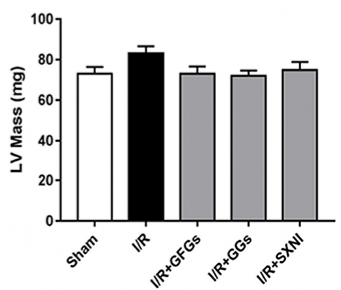

FIGURE 1 | Changes of echocardiographic characterization of cardiac function in myocardial ischemia-reperfusion injury (MIRI) mice. After 30 min of ischemia and $18 \mathrm{~h}$ of reperfusion, the effect of ginkgo flavonol glycosides (GFGs, $2.5 \mathrm{ml} / \mathrm{kg}$ ), ginkgolides (GGs, $2.5 \mathrm{ml} / \mathrm{kg}$ ), and Shuxuening injection (SXNI, $2.5 \mathrm{ml} / \mathrm{kg}$ ) on cardiac function was quantitatively $\mathbf{\Delta}$ evaluated. (A) Representative echocardiography images of different groups. Bar graph quantitation of echocardiographic changes in cardiac function in different groups detected in M-mode: (B) LVEF \%, (C) LVFS \%, (D) CO, (E) stroke volume, (F) LVIDd, (G) LVIDs, (H) LVPWd, (I) LVPWs, (J) LV Vold, (K) LV Vols, (L) heart rate, and (M) LV mass. Values were expressed as mean \pm SEM $(n=5)$. \#\# $P<0.01$ vs. sham group, ${ }^{*} P<0.05$, ${ }^{\star *} P<0.01$ vs. I/R group, $\mathbf{\Delta} P<0.05, \mathbf{\Delta} \mathbf{\Delta} P<0.01$ vs. GFGs group (LV, left ventricular; EF, ejection fraction; FS, LV fractional shortening; CO, cardiac output; LVIDd, LV internal dimensions at diastole; LVIDs, LV internal diameter systole; LVPWd, LV posterior wall diastole; LVPWs, LV posterior wall systole; LV Vols, LV systole volume; LV Vold, LV diastole volume). 
A
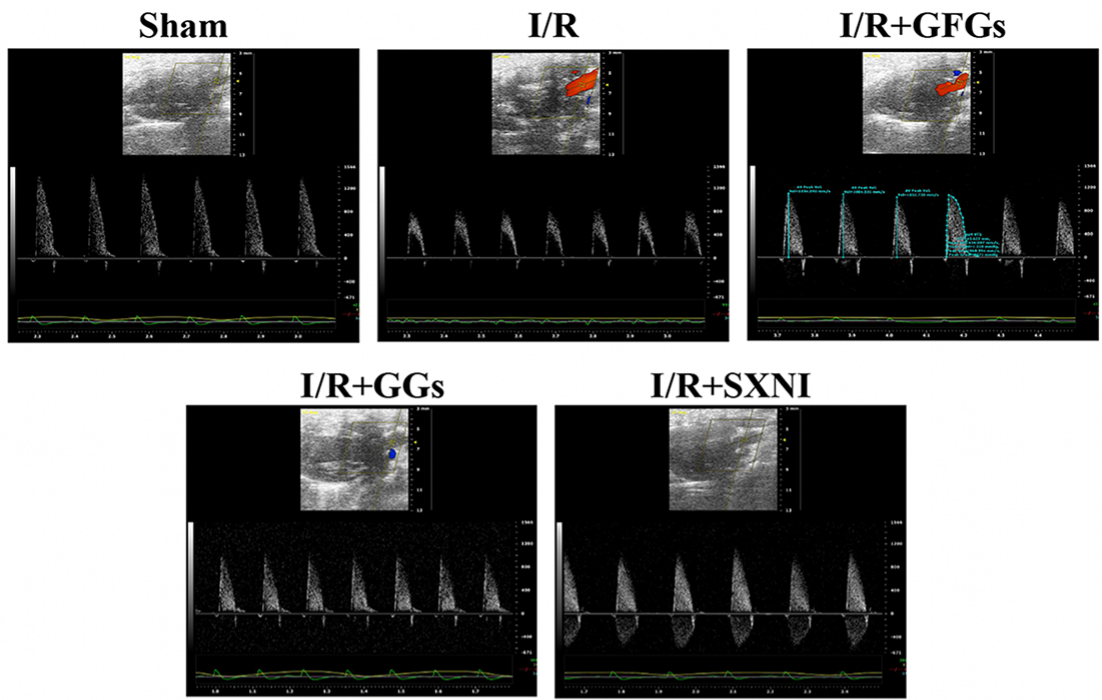

B

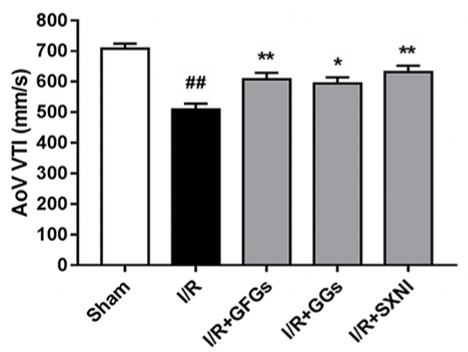

E

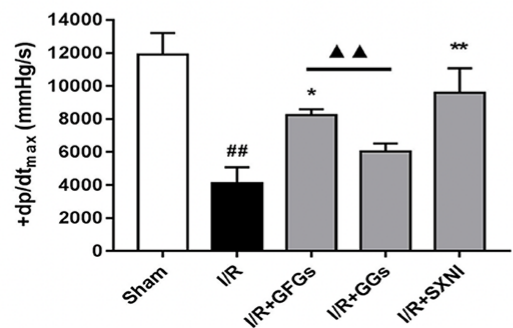

C

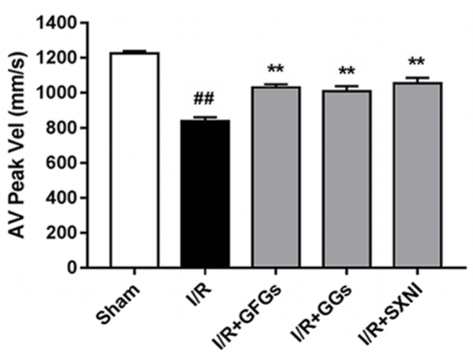

D

2 | GFGs and GGs improved the coronary blood flow and left ventricular function after myocardial ischemia and reperfusion in MIRI mice. After 30 min of ischemia and $18 \mathrm{~h}$ of reperfusion, the effect of GFGs (2.5 ml/kg), GGs (2.5 ml/kg), and SXNI (2.5 mg/kg) on cardiac function was quantitatively evaluated. (A) Representative echocardiography images of coronary blood flow were determined with different groups. (B) AoV VTI, (C) AV peak velocity, and (D) AV peak pressure were detected in color Doppler mode. Quantitative assessment of hemodynamic on left ventricular function based on (E) $+\mathrm{dp} / \mathrm{dt}$ max and (F) $-\mathrm{dp} / \mathrm{dt}$ max Values were expressed as mean \pm SEM $(n=5)$. ${ }^{\# \#} P<0.01$ vs. sham group, ${ }^{*} P<0.05,{ }^{*} P<0.01$ vs. I/R group, $\boldsymbol{\Delta} \mathbf{\Delta} P<0.01$ vs. GFGs group (AV Peak Vel, aortic valve peak velocity; AoV VTI, aorta velocity-time integral mean velocity; $+\mathrm{dp} / \mathrm{dt}_{\max }$, left ventricular maximum upstroke velocity; $-\mathrm{dp} / \mathrm{dt}{ }_{\text {max }}$, left ventricular maximum descent velocity).

damaged tissue (Figure 3A). Therefore, GFGs was more effective than GGs to attenuate myocardial damage in MIRI Mice.

\section{Ginkgolides Are Superior to Ginkgo Flavonol Glycosides in Reducing Cerebral Ischemia-Reperfusion Injury in Cerebral Ischemia-Reperfusion Injury Mice}

The $\mathrm{I} / \mathrm{R}$ injury in middle cerebral artery occlusion (MCAO) mice was estimated via quantifying infarct volumes in TTC- stained brain slices, indirectly calculating the severity of brain edema in micro-CT imaging and neurologic deficit scale. After $24 \mathrm{~h}$ of reperfusion, the cerebral infarct size, the midline offset distance, and neurologic deficit scale in I/R group were significantly increased compared with that of the sham group (Figure 4). GGs and SXNI treatment significantly reduced cerebral infarct size (29.7 and 28.4\%, $p<0.05$ ), whereas GFGs treatment showed a similar trend but did not reach statistical significance (Figure 4A and $\mathrm{C}$ ). Meanwhile, although the shortening of the midline offset distance in GFGs group, which also indirectly proves that the degree of cerebral edema was reduced, 

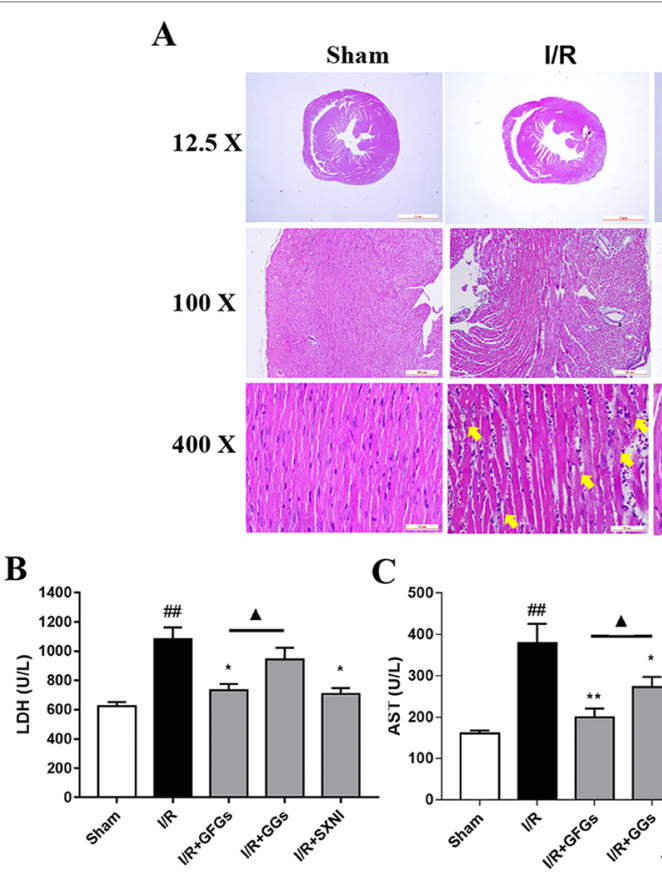

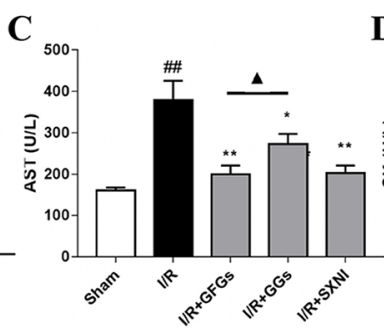

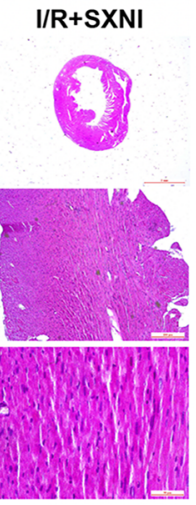

E

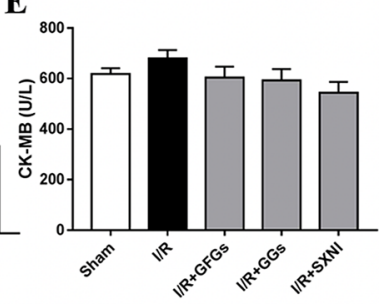

FIGURE 3 | GFGs and GGs alleviated the myocardial I/R injury in MIRI mice. After 30 min of ischemia and $24 \mathrm{~h}$ of reperfusion, the protective effects of GFGs and GGs on MIRI were assessed by hematoxylin and eosin staining and measurement of biochemical parameters. (A) Hematoxylin and eosin staining results for the histopathological of the myocardial tissue indicated the histopathological changes in I/R model as well as that caused by GFGs, GGs, and SXNI. Myocardium damage sections were observed and indicated with yellow arrows in representative pictures (12.5×, 100×, and 400× magnification). (B-E) Effects of GFGs and GGs on the alterations of lactate dehydrogenase (LDH), aspartate aminotransferase (AST), creatine kinase-MB (CK-MB), and creatine kinase (CK) in serum following reperfusion $(n=6)$. Values were expressed as mean \pm SEM. ${ }^{\#} P<0.05,{ }^{\# \#} P<0.01$ vs. sham group, ${ }^{\star} P<0.05,{ }^{* \star} P<0.01$ vs. I/R group, $\Delta P<0.05$ vs. GFGs group.

was evident in the GGs, and SXNI groups, the effects of GGs and SXNI are stronger than those of GFGs (Figure 4B and D). Then, for the protection of neurological function, the therapeutic effects of the GGs and SXNI groups were also stronger than those of the GFGs group (Figure 4E). These results indicated that cerebral protection by SXNI in MCA occlusion mice is attributable mainly to GGs instead of GFGs.

\section{Effects of Ginkgo Flavonol Glycosides and Ginkgolides on the Expression of TWEAK and Fn14 in Myocardial Ischemia- Reperfusion Injury and Cerebral Ischemia- Reperfusion Injury Mice}

The expression levels of TWEAK in heart and brain tissue supernatants of MIRI and CIRI mice were determined by ELISA assay. As shown in Figure 5A and B, TWEAK expressions in model groups (I/R group) were significantly elevated compared with the sham group in MIRI and CIRI mice. Treatment with GFGs, GGs, and SXNI obviously lowered the TWEAK expression in MIRI mice compared with the I/R group, in which GFGs is superior to GGs (Figure 5A). In CIRI mice, GFGs, GGs, and SXNI upregulated TWEAK expression compared with the I/R group. However, the upregulation by GGs and SXNI was significantly higher than that of GFGs (Figure 5B). Meanwhile, Fn14 protein expression level in situ was detected via immunohistochemical staining. In MIRI mice, Fn14 staining was highly elevated in I/R groups, which was markedly reversed in $\mathrm{I} / \mathrm{R}+\mathrm{GFG}$ and $\mathrm{I} / \mathrm{R}+\mathrm{SXNI}$ groups, and to a less content in I/R+GGs groups (Figure 5C). In parallel, the elevated Fn14 expression in I/R group was lowered by the treatment of GFGs, GGs, and SXNI in CIRI mice (Figure 5D). Taken together, these results indicate that in both heart and brain, I/R injury triggers a marked elevation of TWEAKFn14 expression. SXNI oppositely regulated the ligand TWEAK expression in these tissues but universally downregulated the receptor Fn14 in both heart and brain. This SXNI effect is at least in part attributable to that of GFGs or GGs in each tissue, which is consistent with the selective protection effects by GFGs or GGs in heart and brain in MIRI and CIRI mice.

\section{Common and Differential Targeted Genes of Ginkgo Flavonol Glycosides and Ginkgolides in $\mathrm{MICl}$ and $\mathrm{CIRI}$}

Based on previous SXNI transcriptome sequencing data (Lyu et al., 2018), the targeted genes for the GFGs and GGs were identified using IPA. As depicted in Figure 6A, bilobalide, kaempferol, ginkgolide $\mathrm{A}$, and quercetin in SXNI protected the brain from I/R injury in CIRI mice via regulating Calca, Fos, Cdkn1a, Egr1, Mrgprf, Anxa1, Mmp12, and Plaur. Thereinto, bilobalide and ginkgolide A belong to GGs. In addition to the regulation of Selp by ginkgolide B for relieving I/R injury in MIRI mice, the expression of Ptgs2, Sele, 


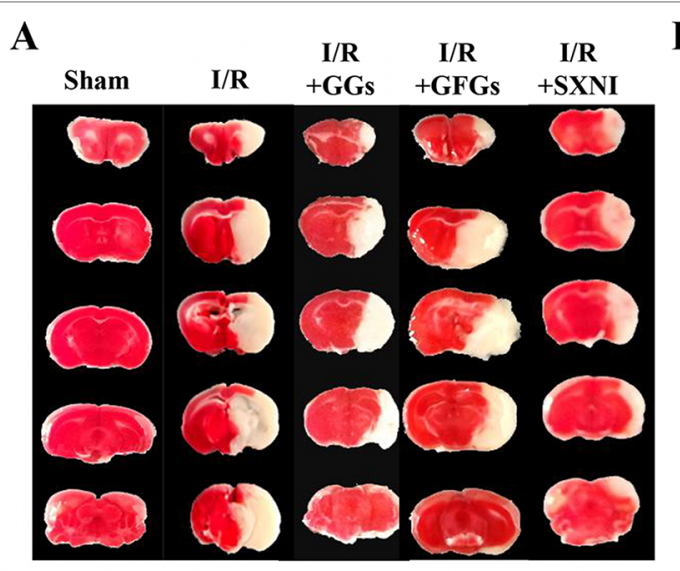

B
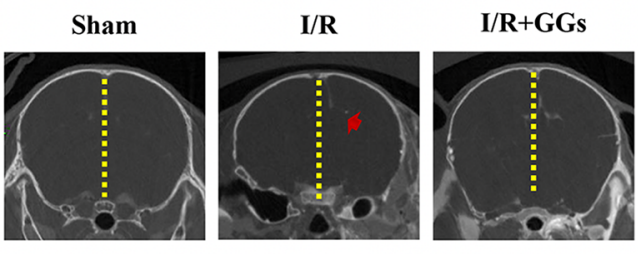

I/R+GFGs

\section{I/R+SXNI}
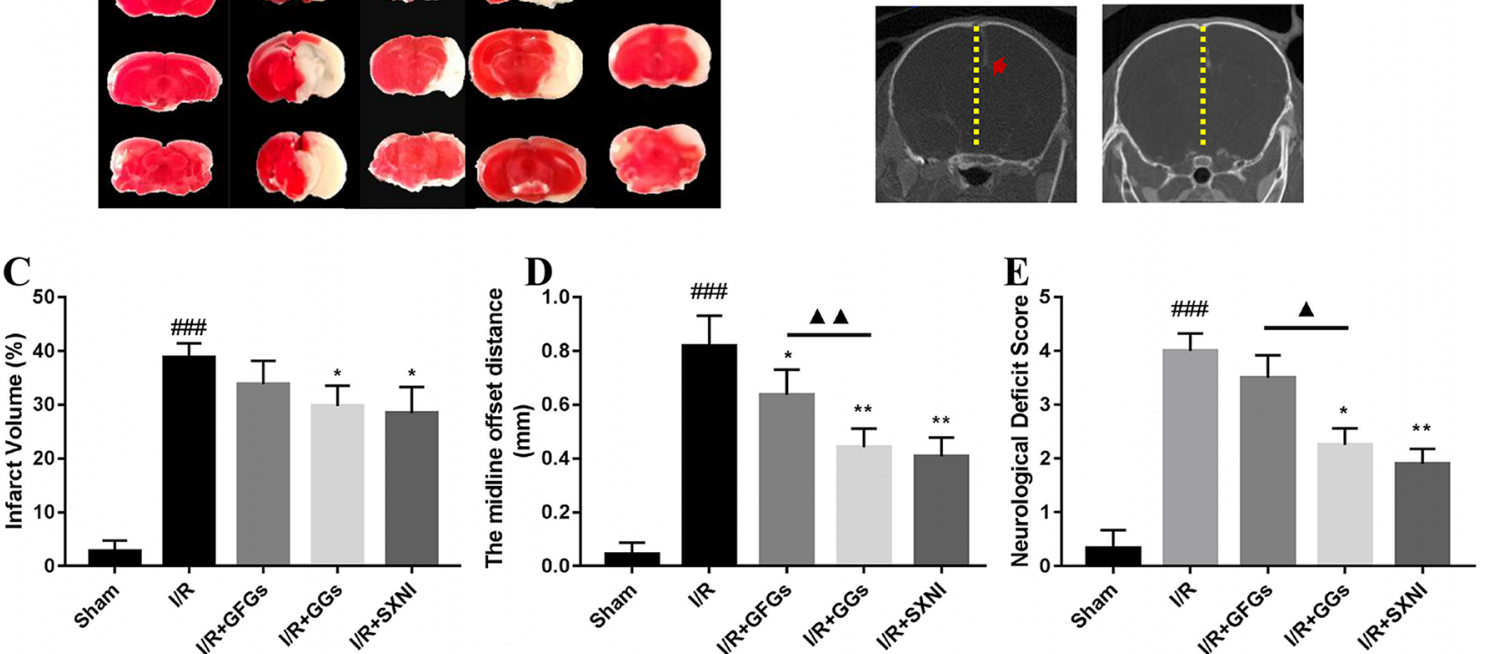

FIGURE 4 | GGs reduced the cerebral infarct size, edema, and neurological deficit score in cerebral ischemia-reperfusion injury (CIRI) mice. After 60 min of ischemia and $24 \mathrm{~h}$ of reperfusion, brain tissue was imaged and transected for detection of cerebral damage by micro- $\mathrm{CT}$ and $2,3,5$-Triphenyl-2H-tetrazolium chloride ( $\mathrm{TTC}$ ) staining, separately. Normal brain tissues were represented in red, whereas the white marked areas of infarct. (A) Representative images of $\Pi \mathrm{C}$ staining in different groups, including sham, I/R model, I/R+GGs, I/R+GFGs, and I/R+SNXI groups. TTC staining was performed at $24 \mathrm{~h}$ after stroke. (C) Quantitation of TTC stain of brain slices as the percentage of infarct volumes of each group $(n=4-5)$. The occurrence of cerebral edema causes the midline of the coronal image of the brain to shift (the red arrow in Figure B was the offset midline). Therefore, the more the midline offset distance, the more severe the brain edema. (B) Representative images of micro-CT imaging in different groups, including sham, I/R model, I/R+GGs, I/R+GFGs, and I/R+SNXI groups. (D) Quantitation of midline offset distance of each group $(n=4-5)$. In addition, the mice were scored for neurological deficits. (E) Neurological deficit score. Values were expressed as mean \pm SEM. \#\# $P<0.001$ vs. sham group, ${ }^{\star} P<0.05,{ }^{\star \star} P<0.05$ vs. I/R group, $\boldsymbol{\Delta} P<0.05, \boldsymbol{\Delta} \mathbf{\Delta} P<0.01$ vs. GFGs group.

Cyp1a1, IL6, Star, Acot1, and Selp was affected by GFGs, which include kaempferol, isorhamnetin, astragatin, quercetin, and rutin. Remarkably, Tnfrsf12a (ENSMUSG00000023905) was detected as an overlapping gene between the MIRI and CIRI (Figure 6A) and was directly regulated by quercetin. Therefore, GFGs and GGs may alleviate I/R injury of heart and brain by differentially affecting the expression of Tnfrsf12a in an organ-specific manner in mice. In addition, we also validated and analyzed the targets of differential regulation of GFGs and GGs. The results indicated that GFGs and GGs can also regulate other differential targets that protect MIRI or CIRI. For example, annexin A1 was downregulated in CIRI without significant changes in MIRI (Figure 6B).

\section{DISCUSSION}

SXNI, consisting of mainly GFGs and GGs, has the characteristics of the synergism of multi-ingredients, multi-targets, and multipathways, producing a superior efficacy over a single drug (Shi et al., 2014; Yang et al., 2017; Lyu et al., 2018). There is an interesting new discovery in this study: the protective effect of
GFGs on MIRI is more significant than that of GGs, while GGs are better than GFGs to reduce CIRI in CIRI mice. The cause of this phenomenon may be connected with the ability of the drug to permeate the blood-brain barrier $(\mathrm{BBB})$. Previous reports showed that terpene trilactones and flavonoid aglycones are more BBB-permeable than glycosides and biflavones (Ma et al., 2014; Konczol et al., 2016). An increased drug concentration in brain may result in a higher pharmacological activity of GGs than GFGs in CIRI mice. Nonetheless, whether the effects of GFGs and GGs on CIRI are related to the BBB-permeability potential should be further verified in the future. However, in another aspect, GFGs may reduce the generation of oxygen-free radicals more than GGs in MIRI mice (Ran et al., 2014; Djouossi et al., 2015). Equally important, although GFGs tend to protect myocardial damage, GGs also play a certain auxiliary role and vice versa.

Furthermore, we also found that GFGs and GGs may relieve both MIRI and CIRI via regulating TWEAK-Fn14 cytokine receptor axis. Interestingly, TWEAK was significantly downregulated by GFGs in MIRI mice and significantly upregulated by GGs in CIRI mice. However, Fn14 was downregulated by GFGs and GGs in both MIRI and CIRI. It has been reported that TWEAK-Fn14 axis is involved 
A

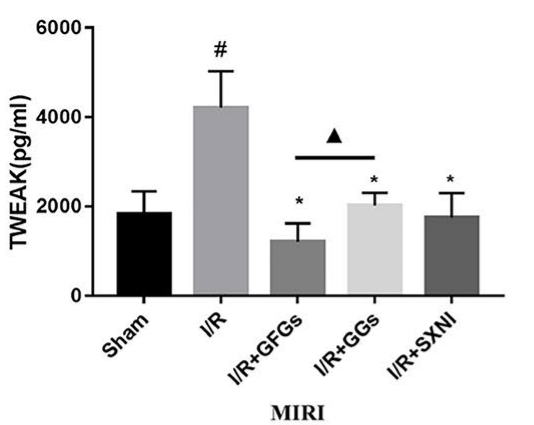

C

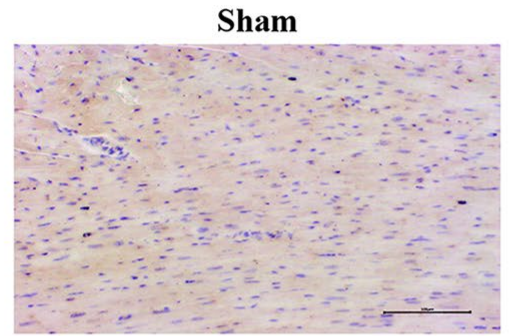

I/R+GGs

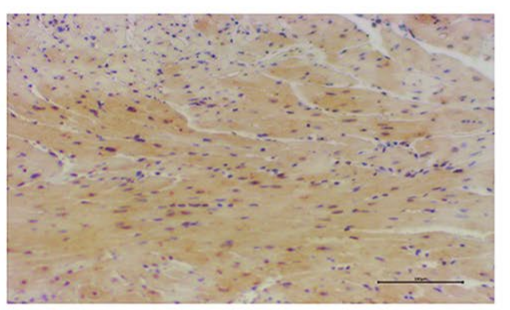

D

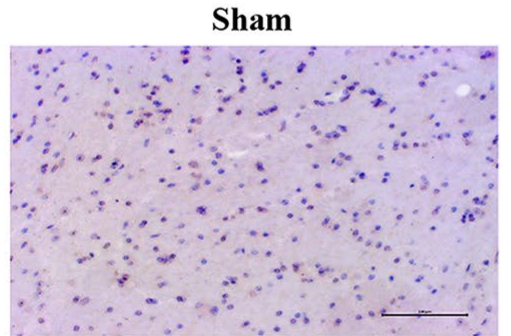

MCAO+GGs

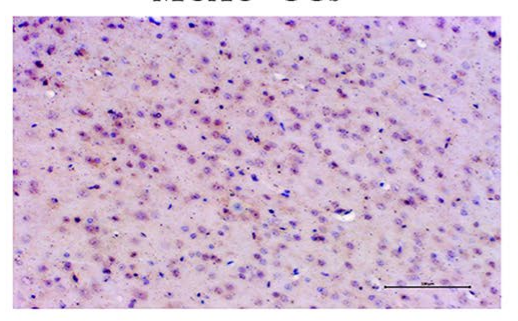

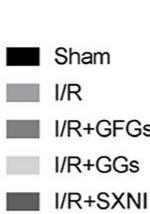

B

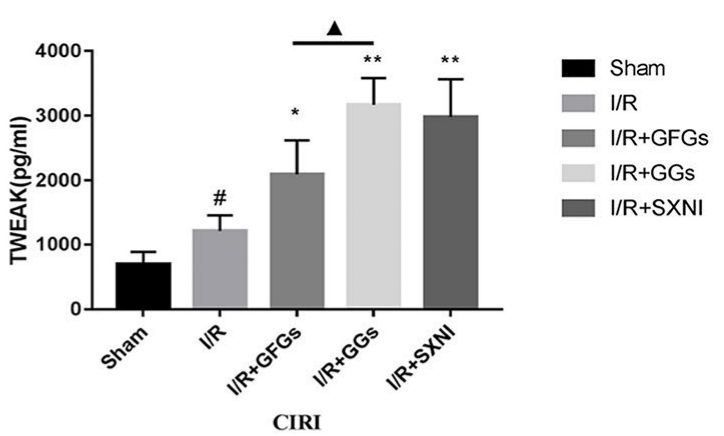

I/R

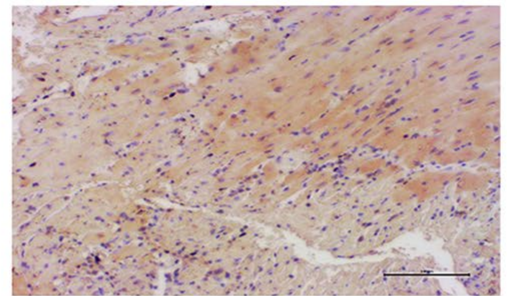

I/R+SXNI

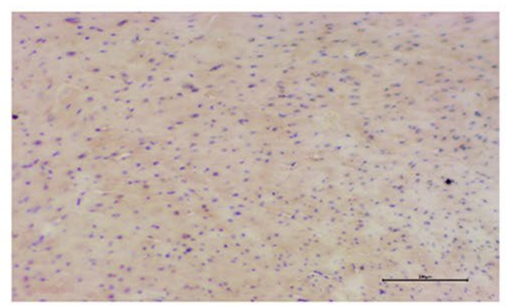

MCAO

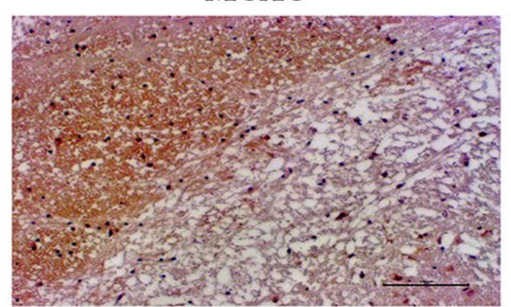

MCAO+SXNI

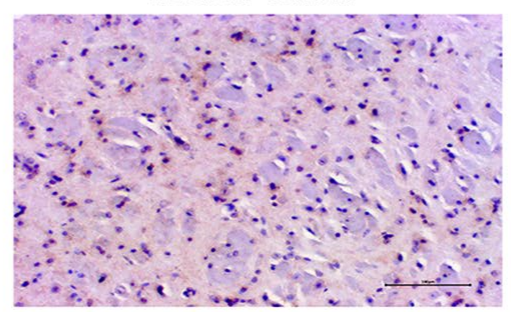

I/R+GFGs
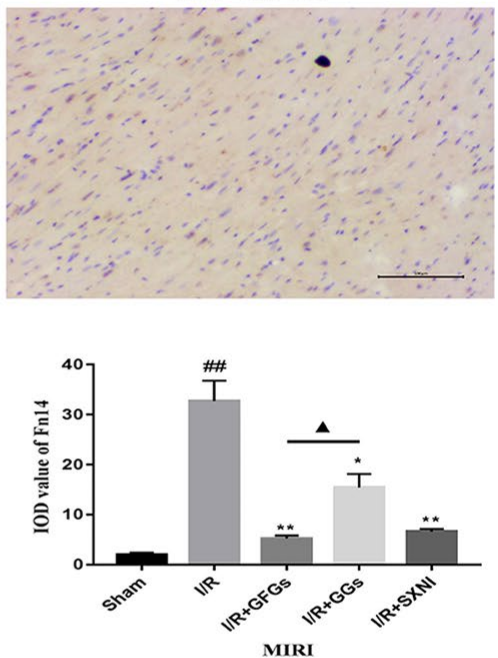

MCAO+GFGs
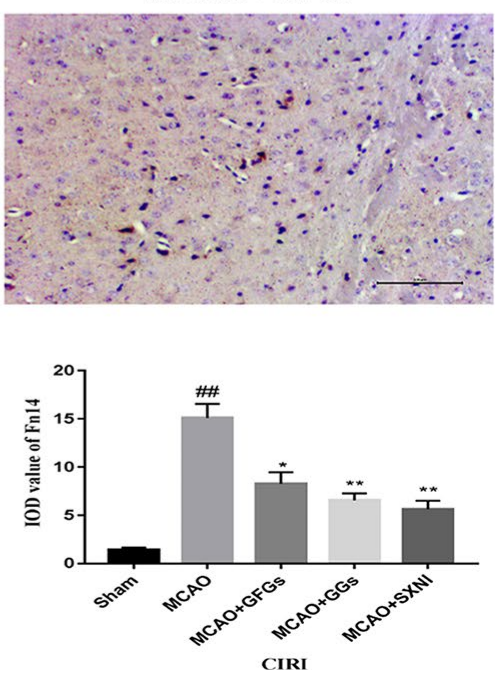

FIGURE 5 | Expression levels of tumor necrosis factor-related weak inducer of apoptosis (TWEAK) and Fn14 in MIRI and CIRI mice. After 24 h of reperfusion, heart and brain were removed from the mice, and their supernatant and tissue block were prepared for immunohistochemical and ELISA analyses of the soluble TWEAK ligand and in situ Fn14 receptor protein, respectively. (A) Effects of GFGs, GGs, and SXNI on the alteration of TWEAK in heart tissue supernatant $(n=3)$. (B) Effects of GFGs, GGs, and SXNI on the alteration of TWEAK in brain tissue supernatant $(n=3)$. (C) Immunohistochemical staining for the heart tissue section of MIRI and control mice indicating the effects of GFGs, GGs, and SXNI on the expression of Fn14 protein (in brown). Representative images (100x magnification) and quantification $(n=3)$ are shown. (D) Immunohistochemical staining for the brain tissue section of CIRI and control mice indicating the effects of GFGs, GGs, and SXNI on the expression of Fn14 protein (in brown). Representative images (100x magnification) and quantification $(n=3)$ are shown. Values were expressed as mean \pm SD B. ${ }^{\#} P<0.01$ vs. sham group, ${ }^{*} P<0.05,{ }^{\star \star} P<0.01$, vs. model group, $\Delta P<0.05$ vs. GFGs group. 
A

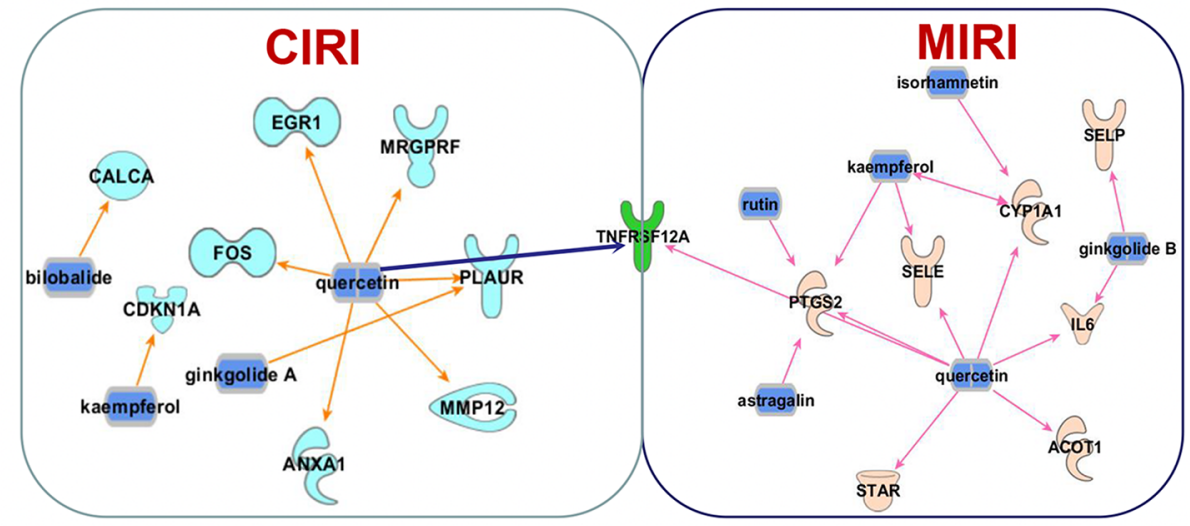

B

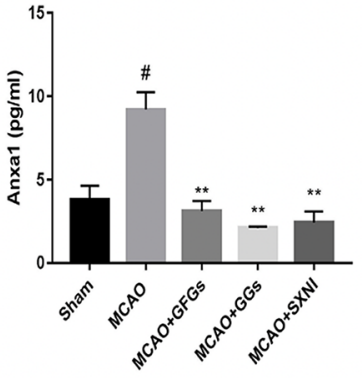

CIRI

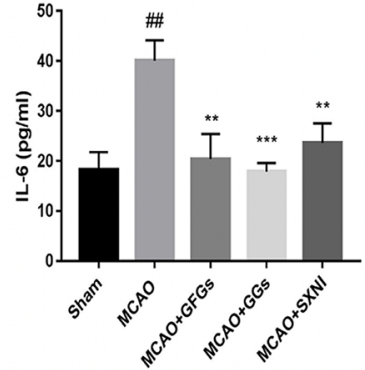

CIRI

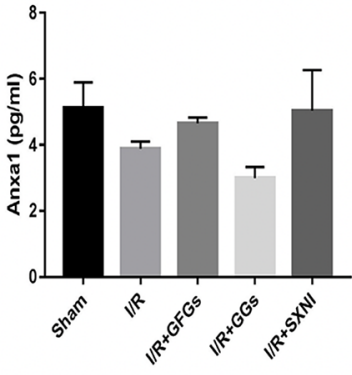

MIRI

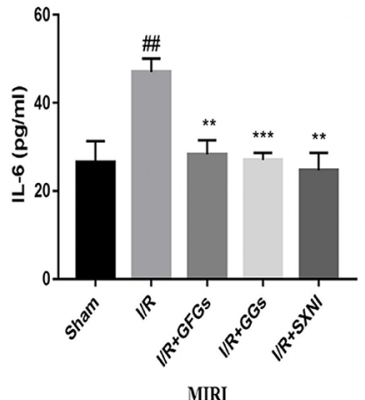

MIRI

FIGURE 6 | GFGs and GGs regulated common and different genes in MIRI and CIRI mice. Previously identified differentially expressed genes, databases of GFGs and GGs of SXNI and their corresponding targets were used to reveal the interaction network by Ingenuity ${ }^{\circledR}$ Pathway Analysis (IPA). (A) The relationship between GFGs and GGs of SXNI (in blue) and their crucially targeted genes in CIRI (in light blue) and MIRI (in light purple), as well as the shared target gene (in green).

(B) Effects of GFGs and GGs on the expression of interleukin 6 and annexin A1 in mice subjected to MIRI or CIRI ( $n=3$ ). Values were expressed as mean \pm SD. ${ }^{\#} P<0.05$, ${ }^{\#} P<0.01$ vs. sham group, ${ }^{\star *} P<0.01$, vs. model group.

in the pathogenesis of stroke via mediating neuronal apoptosis and breakdown of the BBB (Potrovita et al., 2004; Polavarapu et al., 2005; Yepes et al., 2005; Zhang et al., 2007; Haile et al., 2010). Both TWEAK and Fn14 in the ischemic tissue are upregulated in human ischemic stroke and CIRI mice (Potrovita et al., 2004; Yepes et al., 2005; Inta et al., 2008). Our results confirmed this conclusion (Figure 5A and B). Similarly, acute myocardial infarction elevates the level of TWEAK in serum in patients (Chorianopoulos et al., 2010). Recent studies have presented evidence that TWEAK plays a dual role in protecting brain damage, that is, a transient expression of TWEAK may be beneficial to alleviate the damage, but a sustained expression would worsen the situation (Burkly et al., 2007; Winkles, 2008; Mustonen et al., 2010). It has also been shown that TWEAK-Fn14 pathway is critical for the development of hypoxic-ischemic brain injury in immature animal in a genderdependent matter. Specifically, Fn14 gene knockout is beneficial for females, whereas reduced Fn14 expression exacerbated brain injury in males (Kichev et al., 2018). Since our study only used male mice, it remains to be tested if the discordant expression of TWEAK and Fn14 in response to SXNI treatment is attributed to the sex difference. Finally, our network pharmacological analysis suggested that quercetin may directly act on TNFRSF12, a common target of both MIRI and CIRI, to protect against I/R injury. It is worth to further verify the role of quercetin in MIRI and CIRI and explore its interaction with TWEAK-Fn14 pathway.

In previous studies and this study, we predicted and verified that the TWEAK-Fn14 axis as a common target of SXNI for protection of both MIRI and CIRI (Lyu et al., 2018). However, due to the multi-target and multi-pathway characteristics of TCM, SXNI components other than GFGs and GGs may act on other important targets and pathways to alleviate MIRI or CIRI. The continuous exploration of the pharmacological effects of EGB may provide guidance and reference for the clinical application of SXNI and contribute to further understand the pharmacological mechanisms of SXNI.

\section{OTHER LIMITATION AND FUTURE DIRECTIONS}

Our current study investigated the heart and brain locally in MIRI and CIRI models separately. However, we have shown recently 
that CIRI could impact the heart in a model of brain-heart syndrome and another compound Chinese medicine, Danhong injection, effectively protected the heart in this model via local and remote $\beta$-AR signaling (Orgah et al., 2018). Whether SXNI and its GFG/GG components could exert a similar effect remains to be tested.

In this study, we focused on TWEAK-Fn14 axis, which was revealed as the key commonly regulated signaling pathway by SXNI in MIRI and CIRI (Lyu et al., 2018). However, our transcriptome analysis also identified differentially expressed genes by SXNI in heart and brain in an ischemia setting. For example, CYP1A1 and MMP12 may also contribute in the protective effects of GFGs and GGs in MIRI and CIRI (Lv et al., 2008; Ganesan et al., 2010; Mahdessian et al., 2017; Wilsher et al., 2017). We will further explore the roles of GFGs and GGs in regulating these differentially regulated genes and their contribution to the organ-specific protection effects in the future.

\section{CONCLUSION}

We reveal for the first time the different advantages of GFGs and GGs of SXNI in protecting MIRI and CIRI, where GFGs tend to protect myocardial damage while GGs tend to relieve brain injury. Moreover, GFGs may mitigate the I/R injury of the heart in MIRI mice via the downregulated TWEAK-Fn14 axis, whereas GGs may upregulate the signaling pathway to protect the brain from I/R injury in CIRI mice. Overall, the study further elaborates on the material basis and mechanism of SXNI in the treatment of ischemic stroke and coronary heart disease.

\section{DATA AVAILABILITY STATEMENT}

All datasets generated for this study are included in the manuscript and the supplementary files.

\section{REFERENCES}

Abete, P., Della-Morte, D., Gargiulo, G., Basile, C., Langellotto, A., Galizia, G., et al. (2014). Cognitive impairment and cardiovascular diseases in the elderly. A heart-brain continuum hypothesis. Ageing Res. Rev. 18, 41-52. doi: 10.1016/j. arr.2014.07.003

Ago, T., Kuroda, J., Pain, J., Fu, C., Li, H., and Sadoshima, J. (2010). Upregulation of Nox4 by hypertrophic stimuli promotes apoptosis and mitochondrial dysfunction in cardiac myocytes. Circ Res. 106 (7), 1253-1264. doi: 10.1161/ CIRCRESAHA.109.213116

Bederson, J. B., Pitts, L. H., Germano, S. M., Nishimura, M. C., Davis, R. L., and Bartkowski, H. M. (1986). Evaluation of 2,3,5-triphenyltetrazolium chloride as a stain for detection and quantification of experimental cerebral infarction in rats. Stroke 17 (6), 1304-1308. doi: 10.1161/01.STR.17.6.1304

Benjamin, E. J., Virani, S. S., Callaway, C. W., Chamberlain, A. M., Chang, A. R., Cheng, S., et al. (2018). Heart disease and stroke statistics-2018 update: a report from the american heart association. Circulation 137 (12), e67-e492. doi: 10.1161/CIR.0000000000000558

Blanco-Colio, L. M. (2014). TWEAK/Fn14 Axis: a promising target for the treatment of cardiovascular diseases. Front. Immunol. 5, 3. doi: 10.3389/ fimmu.2014.00003

\section{ETHICS STATEMENT}

This study was performed following the recommendations in the Guidance for the Care and Use of Laboratory Animals issued by the Ministry of Science and Technology of China (permit number: TCM-LAEC2014004).The experimental procedures were based on the Directive 2010/63 / EU adopted by the European Union (EU), and all animals were administrated in accordance with the guidelines of Tianjin University of TCM Animal Research Committee (TCM-LAEC2014004).

\section{AUTHOR CONTRIBUTIONS}

YZ contributed to the conception and design of the study. ML, $\mathrm{JN}$, and LL carried out the myocardial ischemia-perfusion correlative experiments provided in Figures 1-3. GX and YW carried out the cerebral ischemia-perfusion assay depicted in Figure 4. GX, SH, and XL participated in the relevant experiments in Figure 5. GX and ML performed the IPA analysis prepared in Figure 6. GF, XW, JH, and XG helped with the design of the study and explanation of results. YZ, GX, and ML wrote the manuscript. All authors reviewed and approved the manuscript.

\section{FUNDING}

This study was supported by the grants from National Science Foundation of China (81873037), Major National Science and Technology Projects (2018YFC1704500) and China Postdoctoral Science Foundation Grant (2019M650989).

\section{ACKNOWLEDGMENTS}

We thank the lab members, particularly Drs. Yuxin Feng, Jian Yang, Rui Shao, and Pengzhi Dong, for stimulating discussion and sharing of reagents.

Bossen, C., Ingold, K., Tardivel, A., Bodmer, J. L., Gaide, O., Hertig, S., et al. (2006). Interactions of tumor necrosis factor (TNF) and TNF receptor family members in the mouse and human. J. Biol. Chem. 281 (20), 13964-13971. doi: 10.1074/ jbc.M601553200

Burkly, L. C., Michaelson, J. S., Hahm, K., Jakubowski, A., and Zheng, T. S. (2007). TWEAKing tissue remodeling by a multifunctional cytokine: role of TWEAK/ Fn14 pathway in health and disease. Cytokine 40 (1), 1-16. doi: 10.1016/j. cyto.2007.09.007

Chen, J., Cao, W., Asare, P. F., Lv, M., Zhu, Y., Li, L., et al. (2016). Amelioration of cardiac dysfunction and ventricular remodeling after myocardial infarction by danhong injection are critically contributed by anti-TGF-beta-mediated fibrosis and angiogenesis mechanisms. J. Ethnopharmacol. 194, 559-570. doi: 10.1016/j.jep.2016.10.025

Chen, J. R., Wei, J., Wang, L. Y., Zhu, Y., Li, L., Olunga, M. A., et al. (2015). Cardioprotection against ischemia/reperfusion injury by QiShenYiQi Pill(R) via ameliorate of multiple mitochondrial dysfunctions. Drug Des. Devel. Ther. 9, 3051-3066. doi: 10.2147/DDDT.S82146

Chen, K., Sun, W., Jiang, Y., Chen, B., Zhao, Y., Sun, J., et al. (2017b). Ginkgolide B Suppresses TLR4-Mediated Inflammatory Response by Inhibiting the Phosphorylation of JAK2/STAT3 and p38 MAPK in High Glucose-Treated HUVECs. Oxid. Med. Cell. Longev. 2017, 9371602. doi: 10.1155/2017/9371602 
Chen, Z., Venkat, P., Seyfried, D., Chopp, M., Yan, T., and Chen, J. (2017c). Brain heart interaction: cardiac complications after stroke. Circ Res. 121 (4), 451-468. doi: 10.1161/CIRCRESAHA.117.311170

Chicheportiche, Y., Bourdon, P. R., Xu, H., Hsu, Y. M., Scott, H., Hession, C., et al. (1997). TWEAK, a new secreted ligand in the tumor necrosis factor family that weakly induces apoptosis. J. Biol. Chem. 272 (51), 32401-32410. doi: 10.1074/ jbc.272.51.32401

Chorianopoulos, E., Jarr, K., Steen, H., Giannitsis, E., Frey, N., and Katus, H. A. (2010). Soluble TWEAK is markedly upregulated in patients with ST-elevation myocardial infarction and related to an adverse short-term outcome. Atherosclerosis 211 (1), 322-326. doi: 10.1016/j.atherosclerosis.2010.02.016

Das, N. A., Carpenter, A. J., Yoshida, T., Kumar, S. A., Gautam, S., Mostany, R., et al. (2018). TRAF3IP2 mediates TWEAK/TWEAKR-induced pro-fibrotic responses in cultured cardiac fibroblasts and the heart. J. Mol. Cell. Cardiol. 121, 107-123. doi: 10.1016/j.yjmcc.2018.07.003

Djouossi, M. G., Tamokou, J. D., Ngnokam, D., Kuiate, J. R., Tapondjou, L. A., Harakat, D., et al. (2015). Antimicrobial and antioxidant flavonoids from the leaves of Oncoba spinosa Forssk. (Salicaceae). BMC Complement Altern. Med. 15, 134. doi: 10.1186/s12906-015-0660-1

Enogieru, A. B., Haylett, W., Hiss, D. C., Bardien, S., and Ekpo, O. E. (2018). Rutin as a Potent Antioxidant: implications for Neurodegenerative Disorders. Oxid. Med. Cell. Longev. 2018, 6241017. doi: 10.1155/2018/6241017

Fadda, L. M., Attia, H. A., Al-Rasheed, N. M., Ali, H. M., and Al-Rasheed, N. M. (2018). Roles of some antioxidants in modulation of cardiac myopathy induced by sodium nitrite via down-regulation of mRNA expression of NF-kappaB, Bax, and flt-1 and suppressing DNA damage. Saudi. Pharm. J. 26 (2), 217-223. doi: 10.1016/j.jsps.2017.12.008

Ganesan, S., Faris, A. N., Comstock, A. T., Chattoraj, S. S., Chattoraj, A., Burgess, J. R., et al. (2010). Quercetin prevents progression of disease in elastase/LPS-exposed mice by negatively regulating MMP expression. Respir. Res. 11 (1), 131. doi: 10.1186/1465-9921-11-131

Gao, E., Lei, Y. H., Shang, X., Huang, Z. M., Zuo, L., Boucher, M., et al. (2010). A novel and efficient model of coronary artery ligation and myocardial infarction in the mouse. Circ. Res. 107 (12), 1445-1453. doi: 10.1161/ CIRCRESAHA.110.223925

Gladstone, D. J., Spring, M., Dorian, P., Panzov, V., Thorpe, K. E., Hall, J., et al. (2014). Atrial fibrillation in patients with cryptogenic stroke. N. Engl. J. Med. 370 (26), 2467-2477. doi: 10.1056/NEJMoa1311376

Goldfine, A. B., and Shoelson, S. E. (2017). Therapeutic approaches targeting inflammation for diabetes and associated cardiovascular risk. J. Clin. Invest 127 (1), 83-93. doi: 10.1172/JCI88884

Granger, D. N., and Kvietys, P. R. (2017). Reperfusion therapy-What's with the obstructed, leaky and broken capillaries? Pathophysiology 24 (4), 213-228. doi: 10.1016/j.pathophys.2017.09.003

Guan, F., Wang, L., Hao, S., Wu, Z., Bai, J., Kang, Z., et al. (2017). Retinol dehydrogenase-10 promotes development and progression of human glioma via the TWEAK-NF- $\kappa B$ axis. Oncotarget 8 (62), 105262-105275. doi: 10.18632/ oncotarget.22166

Guo, M., Suo, Y., Gao, Q., Du, H., Zeng, W., Wang, Y., et al. (2015). The protective mechanism of Ginkgolides and Ginkgo flavonoids on the TNF-alpha induced apoptosis of rat hippocampal neurons and its mechanisms in vitro. Heliyon 1 (1), e00020. doi: 10.1016/j.heliyon.2015.e00020

Gursoy-Ozdemir, Y., Yemisci, M., and Dalkara, T. (2012). Microvascular protection is essential for successful neuroprotection in stroke. J. Neurochem. 123 Suppl 2, 2-11. doi: 10.1111/j.1471-4159.2012.07938.x

Haile, W. B., Echeverry, R., Wu, F., Guzman, J., An, J., Wu, J., et al. (2010). Tumor necrosis factor-like weak inducer of apoptosis and fibroblast growth factor-inducible 14 mediate cerebral ischemia-induced poly(ADP-ribose) polymerase-1 activation and neuronal death. Neuroscience 171 (4), 1256-1264. doi: 10.1016/j.neuroscience.2010.10.029

Han, J. Y., Li, Q., Ma, Z. Z., and Fan, J. Y. (2017). Effects and mechanisms of compound Chinese medicine and major ingredients on microcirculatory dysfunction and organ injury induced by ischemia/reperfusion. Pharmacol. Ther. 177, 146-173. doi: 10.1016/j.pharmthera.2017.03.005

Hao, L., Ren, M., Rong, B., Xie, F., Lin, M. J., Zhao, Y. C., et al. (2018). TWEAK/ Fn14 mediates atrial-derived HL-1 myocytes hypertrophy via JAK2/STAT3 signalling pathway. J. Cell Mol. Med. 22 (9), 4344-4353. doi: 10.1111/ jcmm. 13724
Hu, G., Zeng, W., and Xia, Y. (2017). TWEAK/Fn14 signaling in tumors. Tumour Biol. 39 (6), 1010428317714624. doi: 10.1177/1010428317714624

Hu, Q., Shen, P., Bai, S., Dong, M., Liang, Z., Chen, Z., et al. (2018). Metaboliterelated antidepressant action of diterpene ginkgolides in the prefrontal cortex. Neuropsychiatr. Dis. Treat. 14, 999-1011. doi: 10.2147/NDT.S161351

Iadecola, C., and Anrather, J. (2011). The immunology of stroke: from mechanisms to translation. Nat. Med. 17 (7), 796-808. doi: 10.1038/nm.2399

Inta, I., Frauenknecht, K., Dorr, H., Kohlhof, P., Rabsilber, T., Auffarth, G. U., et al. (2008). Induction of the cytokine TWEAK and its receptor Fn14 in ischemic stroke. J. Neurol. Sci. 275 (1-2), 117-120. doi: 10.1016/j.jns.2008.08.005

Ishikawa, H., Tajiri, N., Vasconcellos, J., Kaneko, Y., Mimura, O., Dezawa, M., et al. (2013). Ischemic stroke brain sends indirect cell death signals to the heart. Stroke 44 (11), 3175-3182. doi: 10.1161/STROKEAHA.113.001714

Jin, G., He, X. R., and Chen, L. P. (2014). The protective effect of ginko bilboa leaves injection on the brain dopamine in the rat model of cerebral ischemia/reperfusion injury. Afr. Health Sci. 14 (3), 725-728. doi: 10.4314/ ahs.v14i3.31

Kichev, A., Baburamani, A. A., Vontell, R., Gressens, P., Burkly, L., Thornton, C., et al. (2018). TWEAK Receptor Deficiency Has Opposite Effects on Female and Male Mice Subjected to Neonatal Hypoxia-Ischemia. Front. Neurol. 9, 230. doi: 10.3389/fneur.2018.00230

Konczol, A., Rendes, K., Dekany, M., Muller, J., Riethmuller, E., and Balogh, G. T. (2016). Blood-brain barrier specific permeability assay reveals N-methylated tyramine derivatives in standardised leaf extracts and herbal products of Ginkgo biloba. J. Pharm. Biomed. Anal. 131, 167-174. doi: 10.1016/j.jpba.2016.08.032

Li, F., Fan, X., Zhang, Y., Pang, L., Ma, X., Song, M., et al. (2016). Cardioprotection by combination of three compounds from ShengMai preparations in mice with myocardial ischemia/reperfusion injury through AMPK activation-mediated mitochondrial fission. Sci. Rep. 6, 37114. doi: 10.1038/srep37114

Li, Y., Liu, J., Liu, Y., Yang, X., Huang, B., and Chen, M. (2017). Inhibitory effect of Ginkgol C17:1 on the biological behavior of tumor cells. Oncol. Lett. 13 (3), 1873-1879. doi: 10.3892/ol.2017.5664

Liao, H. J., Zheng, Y. F., Li, H. Y., and Peng, G. P. (2011). Two new ginkgolides from the leaves of Ginkgo biloba. Planta. Med. 77 (16), 1818-1821. doi: 10.1055/s-0030-1271153

Liu, J., Peng, L., Liu, Y., Wu, K., Wang, S., Wang, X., et al. (2018). Topical TWEAK Accelerates Healing of Experimental Burn Wounds in Mice. Front. Pharmacol. 9, 660. doi: 10.3389/fphar.2018.00660

Lv, X., Wan, J., Yang, J., Cheng, H., Li, Y., Ao, Y., et al. (2008). Cytochrome P450 omega-hydroxylase inhibition reduces cardiomyocyte apoptosis via activation of ERK1/2 signaling in rat myocardial ischemia-reperfusion. Eur. J. Pharmacol. 596 (1-3), 118-126. doi: 10.1016/j.ejphar.2008.08.008

Lyu, M., Cui, Y., Zhao, T., Ning, Z., Ren, J., Jin, X., et al. (2018). Tnfrsf12aMediated Atherosclerosis Signaling and Inflammatory Response as a Common Protection Mechanism of Shuxuening Injection Against Both Myocardial and Cerebral Ischemia-Reperfusion Injuries. Front. Pharmacol. 9, 312. doi: 10.3389/fphar.2018.00312

Lyu, M., Yan, C. L., Liu, H. X., Wang, T. Y., Shi, X. H., Liu, J. P., et al. (2017). Network pharmacology exploration reveals endothelial inflammation as a common mechanism for stroke and coronary artery disease treatment of Danhong injection. Sci. Rep. 7 (1), 15427. doi: 10.1038/s41598-017-14692-3

Ma, H., Li, J., An, M., Gao, X. M., and Chang, Y. X. (2018). A powerful on line ABTS(+)-CE-DAD method to screen and quantify major antioxidants for quality control of Shuxuening Injection. Sci. Rep. 8 (1), 5441. doi: 10.1038/ s41598-018-23748-X

Ma, S., Liu, X., Xu, Q., and Zhang, X. (2014). Transport of ginkgolides with different lipophilicities based on an hCMEC/D3 cell monolayer as a bloodbrain barrier cell model. Life Sci. 114 (2), 93-101. doi: 10.1016/j.lfs.2014.08.006

Mahdessian, H., Perisic Matic, L., Lengquist, M., Gertow, K., Sennblad, B., Baldassarre, D., et al. (2017). Integrative studies implicate matrix metalloproteinase-12 as a culprit gene for large-artery atherosclerotic stroke. J. Intern. Med. 282 (5), 429-444. doi: 10.1111/joim.12655

Marsters, S. A., Sheridan, J. P., Pitti, R. M., Brush, J., Goddard, A., and Ashkenazi, A. (1998). Identification of a ligand for the death-domain-containing receptor Apo3. Curr. Biol. 8 (9), 525-528. doi: 10.1016/S0960-9822(98)70204-0

Marunaka, Y., Marunaka, R., Sun, H., Yamamoto, T., Kanamura, N., Inui, T., et al. (2017). Actions of quercetin, a polyphenol, on blood pressure. Molecules 22 (2), 209. doi: 10.3390/molecules22020209 
Mehrbod, P., Abdalla, M. A., Fotouhi, F., Heidarzadeh, M., Aro, A. O., Eloff, J. N., et al. (2018). Immunomodulatory properties of quercetin-3-O-alpha-Lrhamnopyranoside from Rapanea melanophloeos against influenza a virus. BMC Complement Altern. Med. 18 (1), 184. doi: 10.1186/s12906-018-2246-1

Meighan-Mantha, R. L., Hsu, D. K. W., Guo, Y., Brown, S. A. N., Feng, S. L. Y., Peifley, K. A., et al. (1999). The mitogen-inducible Fn14 gene encodes a type I transmembrane protein that modulates fibroblast adhesion and migration. J. Biol. Chem. 274, 33166-33176. doi: 10.1074/jbc.274.46.33166

Mohanta, T. K., Tamboli, Y., and Zubaidha, P. K. (2014). Phytochemical and medicinal importance of Ginkgo biloba L. Nat. Prod. Res. 28 (10), 746-752. doi: 10.1080/14786419.2013.879303

Muntean, D. M., Sturza, A., Danila, M. D., Borza, C., Duicu, O. M., and Mornos, C. (2016). The role of mitochondrial reactive oxygen species in cardiovascular injury and protective strategies. Oxid. Med. Cell. Longev. 2016, 8254942. doi: $10.1155 / 2016 / 8254942$

Mustonen, E., Sakkinen, H., Tokola, H., Isopoussu, E., Aro, J., Leskinen, H., et al. (2010). Tumour necrosis factor-like weak inducer of apoptosis (TWEAK) and its receptor Fn14 during cardiac remodelling in rats. Acta Physiol. (Oxf) 199 (1), 11-22. doi: 10.1111/j.1748-1716.2010.02080.x

Orgah, J. O., Yu, J., Zhao, T., Wang, L., Yang, M., Zhang, Y., et al. (2018). Danhong injection reversed cardiac abnormality in brain-heart syndrome via local and remote $\beta$-adrenergic receptor signaling. Front. Pharmacol. 9, 692. doi: 10.3389/ fphar.2018.00692

Park, J. Y., Lee, S. K., Kim, J. Y., Je, K. H., Schellingerhout, D., and Kim, D. E. (2014). A new micro-computed tomography-based high-resolution bloodbrain barrier imaging technique to study ischemic stroke. Stroke 45 (8), 24802484. doi: 10.1161/STROKEAHA.114.006297

Patel, R. V., Mistry, B. M., Shinde, S. K., Syed, R., Singh, V., and Shin, H. S. (2018). Therapeutic potential of quercetin as a cardiovascular agent. Eur. J. Med. Chem. 155, 889-904. doi: 10.1016/j.ejmech.2018.06.053

Patra, A., Satpathy, S., Shenoy, A. K., Bush, J. A., Kazi, M., and Hussain, M. D. (2018). Formulation and evaluation of mixed polymeric micelles of quercetin for treatment of breast, ovarian, and multidrug resistant cancers. Int. J. Nanomedicine. 13, 2869-2881. doi: 10.2147/IJN.S153094

Pei, J., Fu, W., Yang, L., Zhang, Z., and Liu, Y. (2013). Oxidative stress is involved in the pathogenesis of keshan disease (an endemic dilated cardiomyopathy) in china. Oxid. Med. Cell. Longev. 2013, 1-5. doi: 10.1155/2013/474203

Polavarapu, R., Gongora, M. C., Winkles, J. A., and Yepes, M. (2005). Tumor necrosis factor-like weak inducer of apoptosis increases the permeability of the neurovascular unit through nuclear factor-kappa B pathway activation. J. Neurosci. 25 (44), 10094-10100. doi: 10.1523/JNEUROSCI.3382-05.2005

Potrovita, I., Zhang, W., Burkly, L., Hahm, K., Lincecum, J., Wang, M. Z., et al. (2004). Tumor necrosis factor-like weak inducer of apoptosis-induced neurodegeneration. J. Neurosci. 24 (38), 8237-8244. doi: 10.1523/JNEUROSCI. 1089-04.2004

Priyanka, A., Nisha, V. M., Anusree, S. S., and Raghu, K. G. (2014). Bilobalide attenuates hypoxia induced oxidative stress, inflammation, and mitochondrial dysfunctions in 3T3-L1 adipocytes via its antioxidant potential. Free Radic. Res. 48 (10), 1206-1217. doi: 10.3109/10715762.2014.945442

Prosser, J., MacGregor, L., Lees, K. R., Diener, H. C., Hacke, W., Davis, S., et al. (2007). Predictors of early cardiac morbidity and mortality after ischemic stroke. Stroke 38 (8), 2295-2302. doi: 10.1161/STROKEAHA.106.471813

Ran, K., Yang, D. L., Chang, Y. T., Duan, K. M., Ou, Y. W., Wang, H. P., et al. (2014). Ginkgo biloba extract postconditioning reduces myocardial ischemia reperfusion injury. Genet. Mol. Res. 13 (2), 2703-2708. doi: 10.4238/2014. April.8.14

Rousselet, E., Kriz, J., and Seidah, N. G. (2012). Mouse model of intraluminal MCAO: cerebral infarct evaluation by cresyl violet staining. J. Vis. Exp. (69), 4038. doi: $10.3791 / 4038$

Rui, T. Q., Zhang, L., Qiao, H. Z., Huang, P., Qian, S., Li, J. S., et al. (2016). Preparation and physicochemical and pharmacokinetic characterization of ginkgo lactone nanosuspensions for antiplatelet aggregation. J. Pharm. Sci. 105 (1), 242-249. doi: 10.1016/j.xphs.2015.10.002

Saini, A. S., Taliyan, R., and Sharma, P. L. (2014). Protective effect and mechanism of Ginkgo biloba extract-EGb 761 on STZ-induced diabetic cardiomyopathy in rats. Pharmacogn. Mag. 10 (38), 172-178. doi: 10.4103/0973-1296.131031

Samuels, M. A. (2007). The brain-heart connection. Circulation 116 (1), 77-84. doi: 10.1161/CIRCULATIONAHA.106.678995
Sanderson, T. H., Reynolds, C. A., Kumar, R., Przyklenk, K., and Huttemann, M. (2013). Molecular mechanisms of ischemia-reperfusion injury in brain: pivotal role of the mitochondrial membrane potential in reactive oxygen species generation. Mol. Neurobiol. 47 (1), 9-23. doi: 10.1007/ s12035-012-8344-z

Sanna, T., Diener, H. C., Passman, R. S., Di Lazzaro, V., Bernstein, R. A., Morillo, C. A., et al. (2014). Cryptogenic stroke and underlying atrial fibrillation. N. Engl. J. Med. 370 (26), 2478-2486. doi: 10.1056/NEJMoa1313600

Shi, S. H., Cai, Y. P., Cai, X. J., Zheng, X. Y., Cao, D. S., Ye, F. Q., et al. (2014). A network pharmacology approach to understanding the mechanisms of action of traditional medicine: bushenhuoxue formula for treatment of chronic kidney disease. PLoS One 9 (3), e89123. doi: 10.1371/journal.pone. 0089123

Sidler, D., Wu, P., Herro, R., Claus, M., Wolf, D., Kawakami, Y., et al. (2017). TWEAK mediates inflammation in experimental atopic dermatitis and psoriasis. Nat. Commun. 8, 15395. doi: 10.1038/ncomms 15395

Son, A., Oshio, T., Kawamura, Y. I., Hagiwara, T., Yamazaki, M., Inagaki-Ohara, K., et al. (2013). TWEAK/Fn14 pathway promotes a T helper 2-type chronic colitis with fibrosis in mice. Mucosal Immunol. 6 (6), 1131-1142. doi: 10.1038/ mi.2013.10

Song, J. Y., Truong, D. V., and Yang, B. S. (2018). Quercetin shows the pharmacological activity to simultaneously downregulate the inflammatory and fibrotic responses to tissue injury in association with its ability to target multi-kinases. Pharmacology 102 (3-4), 142-153. doi: 10.1159/000490417

van Beek, T. A., and Montoro, P. (2009). Chemical analysis and quality control of Ginkgo biloba leaves, extracts, and phytopharmaceuticals. J. Chromatogr A. 1216 (11), 2002-2032. doi: 10.1016/j.chroma.2009.01.013

Wan, W., Zhang, C., Danielsen, M., Li, Q., Chen, W., Chan, Y., et al. (2016). EGb761 improves cognitive function and regulates inflammatory responses in the APP/PS1 mouse. Exp. Gerontol. 81, 92-100. doi: 10.1016/j. exger.2016.05.007

Wang, L., Yu, J., Fordjour, P. A., Xing, X., Gao, H., Li, Y., et al. (2017). Danshen injection prevents heart failure by attenuating post-infarct remodeling. J. Ethnopharmacol. 205, 22-32. doi: 10.1016/j.jep.2017.04.027

Wiley, S. R., Cassiano, L., Lofton, T., Davissmith, T., Winkles, J. A., Lindner, V., et al. (2001). A novel TNF receptor family member binds TWEAK and is implicated in angiogenesis. Immunity 15 (5), 837-846. doi: 10.1016/ S1074-7613(01)00232-1

Wilsher, N. E., Arroo, R. R., Matsoukas, M. T., Tsatsakis, A. M., Spandidos, D. A., and Androutsopoulos, V. P. (2017). Cytochrome P450 CYP1 metabolism of hydroxylated flavones and flavonols: selective bioactivation of luteolin in breast cancer cells. Food. Chem. Toxicol. 110, 383-394. doi: 10.1016/j.fct.2017. 10.051

Winkles, J. A. (2008). The TWEAK-Fn14 cytokine-receptor axis: discovery, biology and therapeutic targeting. Nat. Rev. Drug. Discov. 7 (5), 411-425. doi: $10.1038 / \mathrm{nrd} 2488$

Xu, R. D., Feng, F., Yu, X. S., Liu, Z. D., and Lao, L. F. (2018). miR-149-5p inhibits cell growth by regulating TWEAK/Fn14/PI3K/AKT pathway and predicts favorable survival in human osteosarcoma. Int. J. Immunopathol. Pharmacol. 32, 2058738418786656. doi: 10.1177/2058738418786656

Xue, L., Liu, L., Huang, J., Wen, J., Yang, R., Bo, L., et al. (2017). Tumor necrosis factor-like weak inducer of apoptosis activates type i interferon signals in lupus nephritis. Biomed. Res. Int. 2017, 4927376. doi: 10.1155/2017/4927376

Yang, Y., Jiang, S., Dong, Y., Fan, C., Zhao, L., Yang, X., et al. (2015). Melatonin prevents cell death and mitochondrial dysfunction via a SIRT1-dependent mechanism during ischemic-stroke in mice. J. Pineal. Res. 58 (1), 61-70. doi: 10.1111/jpi.12193

Yang, Y., Li, Y., Wang, J., Sun, K., Tao, W., Wang, Z., et al. (2017). Systematic investigation of Ginkgo biloba leaves for treating cardio-cerebrovascular diseases in an animal model. ACS. Chem. Biol. 12 (5), 1363-1372. doi: 10.1021/ acschembio.6b00762

Yepes, M., Brown, S. A. N., Moore, E. G., Smith, E. P., Lawrence, D. A., and Winkles, J. A. (2005). A soluble Fn14-Fc decoy receptor reduces infarct volume in a murine model of cerebral ischemia. Am. J. Pathol. 166 (2), 511-520. doi: 10.1016/S0002-9440(10)62273-0

Yu, T., Fan, Y., Xu, Y., Xu, L., Xu, G., Cao, F., et al. (2018). Standardized Ginkgo biloba extract EGb 761(R) attenuates early brain injury following subarachnoid hemorrhage via suppressing neuronal apoptosis through the activation of Akt 
signaling. Biomed. Pharmacother. 107, 329-337. doi: 10.1016/j.biopha.2018. 08.012

Zhang, X., Winkles, J. A., Gongora, M. C., Polavarapu, R., Michaelson, J. S., Hahm, K., et al. (2007). TWEAK-Fn 14 pathway inhibition protects the integrity of the neurovascular unit during cerebral ischemia. J. Cereb Blood Flow Metab. 27 (3), 534-544. doi: 10.1038/sj.jcbfm. 9600368

Zhao, X., Yao, H., Yin, H.-L., Zhu, Q.-L., Sun, J.-L., Ma, W., et al. (2013). Ginkgo biloba Extract and Ginkgolide Antiarrhythmic Potential by Targeting hERG and ICa-L Channel. J. Pharmacol. Sci. 123 (4), 318-327. doi: 10.1254/jphs.13118FP

Zheng, J. L., Li, B. S., Cao, X. C., Zhuo, W. K., and Zhang, G. (2016). Alleviation of spinal cord injury by Ginkgolide B via the inhibition of STAT1 expression. Genet. Mol. Res. 15 (2). doi: 10.4238/gmr.15027673

Zhuang, M., Qiu, H., Li, P., Hu, L., Wang, Y., and Rao, L. (2018). Islet protection and amelioration of type 2 diabetes mellitus by treatment with quercetin from the flowers of Edgeworthia gardneri. Drug Des. Devel. Ther. 12, 955-966. doi: 10.2147/DDDT.S153898

Conflict of Interest Statement: The authors declare that the research was conducted in the absence of any commercial or financial relationships that could be construed as a potential conflict of interest.

Copyright $\odot 2019$ Xiao, Lyu, Wang, He, Liu, Ni, Li, Fan, Han, Gao, Wang and Zhu. This is an open-access article distributed under the terms of the Creative Commons Attribution License (CC BY). The use, distribution or reproduction in other forums is permitted, provided the original author(s) and the copyright owner(s) are credited and that the original publication in this journal is cited, in accordance with accepted academic practice. No use, distribution or reproduction is permitted which does not comply with these terms. 